University of Louisville

ThinkIR: The University of Louisville's Institutional Repository

$5-2018$

\title{
Maturation of human induced pluripotent stem cell derived engineered cardiac tissues using chief transfection and chronic optical pacing.
}

Marc M. Dwenger

University of Louisville

Follow this and additional works at: https://ir.library.louisville.edu/etd

Part of the Cardiovascular Diseases Commons, and the Therapeutics Commons

\section{Recommended Citation}

Dwenger, Marc M., "Maturation of human induced pluripotent stem cell derived engineered cardiac tissues using chief transfection and chronic optical pacing." (2018). Electronic Theses and Dissertations. Paper 2910.

https://doi.org/10.18297/etd/2910

This Master's Thesis is brought to you for free and open access by ThinkIR: The University of Louisville's Institutional Repository. It has been accepted for inclusion in Electronic Theses and Dissertations by an authorized administrator of ThinkIR: The University of Louisville's Institutional Repository. This title appears here courtesy of the author, who has retained all other copyrights. For more information, please contact thinkir@louisville.edu. 


\title{
MATURATION OF HUMAN INDUCED PLURIPOTENT STEM CELL DERIVED ENGINEERED
} CARDIAC TISSUES USING CHIEF TRANSFECTION AND CHRONIC OPTICAL PACING

\author{
By
}

Marc Matthew Dwenger

B.A., Simpson College, 2013

\author{
A Thesis \\ Submitted to the Faculty of the \\ School of Medicine of the University of Louisville \\ In Partial Fulfillment of the Requirements \\ for the Degree of
}

Master of Science in Pharmacology and Toxicology

Department of Pharmacology and Toxicology

University of Louisville

Louisville, Kentucky

May 2018 



\section{MATURATION OF HUMAN INDUCED PLURIPOTENT STEM CELL DERIVED ENGINEERED}

CARDIAC TISSUES USING CHIEF TRANSFECTION AND CHRONIC OPTICAL PACING

By

Marc M. Dwenger

B.A., Simpson College, 2013

A Thesis Approved on

May 25, 2017

by the following Thesis Committee:

Bradley B. Keller, M.D.

William Guido, Ph.D.

Lu Cai, M.D., Ph.D.

Levi Beverly, Ph.D.

Joshua L. Hood, M.D., Ph.D. 


\section{ACKNOWLEDGEMENTS}

I am grateful to everyone that has helped me with this project, especially, my fellow lab members. I am grateful to Dr. Keller for his mentorship and guidance. I am thankful to Dr. Nakane, Dr. Ye, and Dr. Setozaki for generating the h-iPSC-derived cardiomyocytes. I want to thank Joe Tinney for assisting with engineered cardiac tissue generation and force testing. I am grateful to Dr. Yuan for doing the histology. I am thankful to Dr. Kowalski for his alignment analyses and mentorship. I am also thankful to the Guido lab for providing expertise concerning optogenetics and the AAV virus. I am grateful to Peter for doing the patch clamp of the transfected ECTs. I also want to thank Dr. Beverly's lab for helping at the outset of this project. I would also like to thank my friends and family for their support. 


\section{ABSTRACT \\ MATURATION OF HUMAN INDUCED PLURIPOTENT STEM CELL DERIVED ENGINEERED CARDIAC TISSUES USING CHIEF TRANSFECTION AND CHRONIC OPTICAL PACING \\ Marc M. Dwenger}

May 25, 2017

The immaturity of engineered cardiac tissues (ECTs) limits their ability to regenerate damaged myocardium and to serve as in vitro disease models and surrogates for drug toxicity testing. Several chronic biomimetic conditioning protocols, including mechanical stretch, perfusion, and electrical stimulation promote ECT maturation but have significant technical limitations. Non-contacting chronic light stimulation using heterologously expressed light-sensitive ChIEF ion channels, termed optogenetics, may be an advantageous alternative to chronic electrical stimulation. As a proof of principle, we transfected ECTs using an AAV packaged ChIEF and then verified acute optical pacing (OP) by patch clamp. We then chronically OP ECTs for 7 days above the intrinsic beat rate. Chronic OP resulted in improved ECT electrophysiological properties; however, ECT force generation and histology remained unchanged. Some changes in cardiac relevant gene expression were noted. This work validates a novel chronic OP paradigm that can be used to identify strategies for optimal in vitro ECT maturation. 
TABLE OF CONTENTS

PAGE

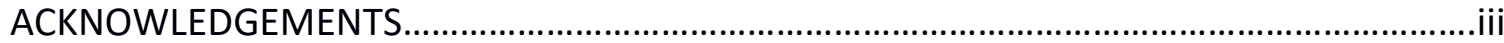

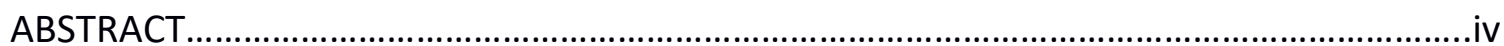

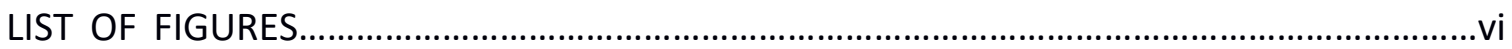

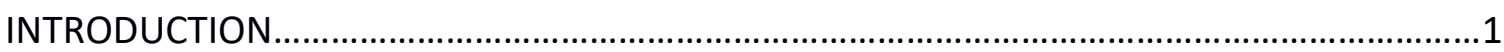

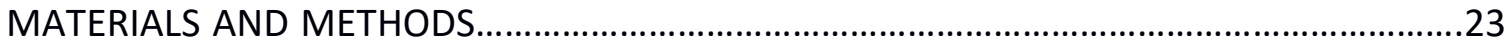

Differentiation of Human iPS cells into Cardiac Cells.............................................23

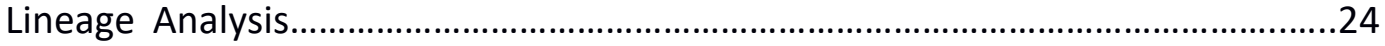

Engineering of ChIEF-Linear Cardiac Tissue..........................................................25

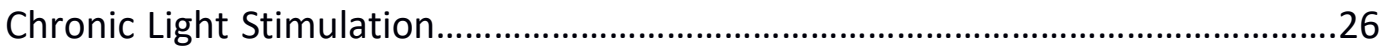

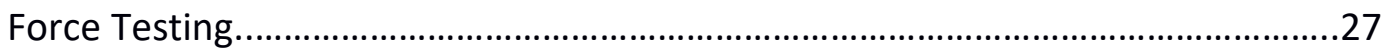

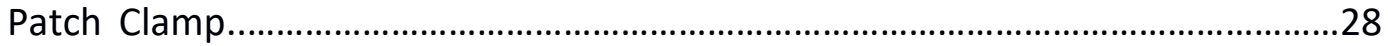

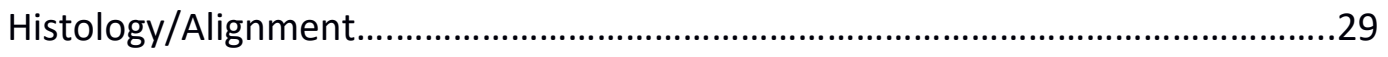

Real-Time Quantitative Polymerase Chain Reaction.................................................31

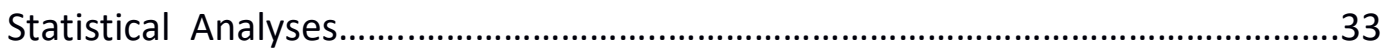

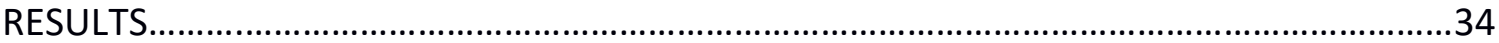

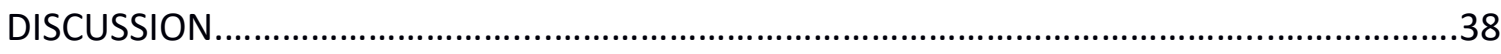

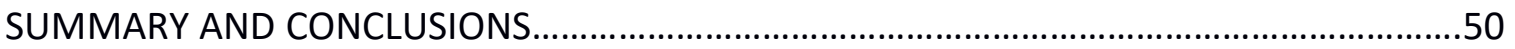

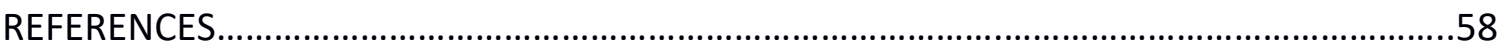

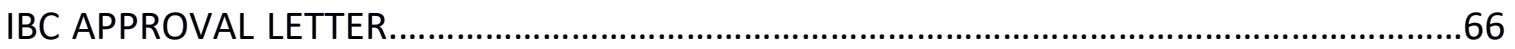

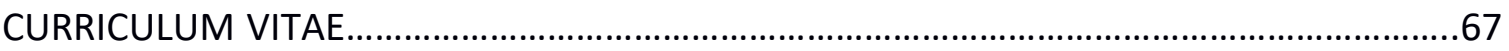




\section{LIST OF FIGURES}

$\begin{array}{ll}\text { FIGURE } & \text { PAGE }\end{array}$

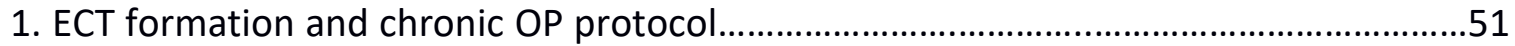

2. Impact of chronic OP on functional maturation of h-iPSC ECTs....................................52

3. Impact of chronic OP on structural maturation of h-iPSC ECTs.......................................53

4. Quantitative PCR comparison between D14 control and chronic OP ECTs....................54

5. Quantitative PCR comparison between D14 control, D28, and chronic OP ECTs..........55

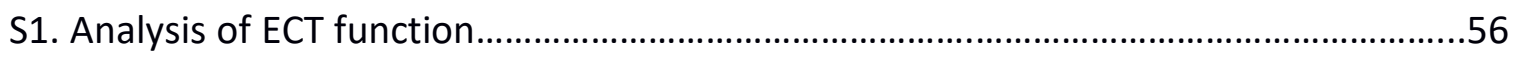

S2. Representative force-length tracing at increasing pacing rates..................................57 


\section{INTRODUCTION}

Heart disease causes many deaths worldwide, and the heart does not fully regenerate after injury. ${ }^{1,2}$ When higher vertebrate heart muscle is damaged, the heart is unable to fully regenerate cardiomyocytes lost to trauma. ${ }^{2}$ This incomplete regeneration leads to pump dysfunction and symptoms of clinical heart failure. ${ }^{3}$ Currently, most therapies do not directly remedy this loss of cardiac ability. ${ }^{3}$ To overcome cardiac tissue's limited regenerative capacity and restore the heart's pumping ability, researchers are developing cell therapy and tissue engineering paradigms. ${ }^{3}$

Cell therapy involves transplantation of healthy cells into the damaged tissue to regenerate the injured myocardium. ${ }^{4}$ The cells regenerate the damaged muscle through many possible mechanisms: formation of new blood vessels, paracrine mechanisms, and cell fusion. ${ }^{4}$ Cell therapy research has utilized many different cell types: embryonic stem cells, skeletal myoblasts, bone-marrow derived stem cells, adipose-derived mesenchymal stem cells, induced pluripotent stem cells, and cardiac stems cells. ${ }^{4}$ These cells have varying efficiencies to differentiate into cardiac cells ex vivo before implantation, or undifferentiated stem cells, transplanted into the heart, become cardiac cells in vivo, regenerating the necrotic tissue. ${ }^{4}$ Cell therapy clinical trials demonstrate improved myocardium function following cell delivery. ${ }^{4}$ However, after cell 
injection, greater than 90 percent of the cells are lost within the first few days, limiting the restorative ability of cell therapy. ${ }^{4}$

Tissue engineering strategies could improve the negative outcomes of cell therapy; instead of isolated cell delivery, scientists combine cells with biomaterials, before transplantation, that increase cell retention and differentiation. ${ }^{5}$ Many different tissue engineering protocols exist such as, cardiac patches, injectable delivery matrices, microspheres for cell encapsulation, and engineered cardiac tissues (ECTs). ${ }^{5,6}$ Cardiac patches involve implantation of cells attached to a biopolymer scaffold; whereas, injectable delivery matrices are thermosensitive materials that gel on injection to improve cell retention. ${ }^{5}$ Microspheres capture individual cells in self assembling spherical constructs for the same purpose.

Engineered cardiac tissues involve the formation of a tissue construct by combination of cells and extracellular matrix. ${ }^{6}$ In this strategy, used by our lab, a mold forms a gelatinous mixture of cells, liquid collagen, Matrigel, and growth supplements into viable cardiac tissue. ${ }^{6,7}$ Surgeons, then, implant the tissue onto the heart to regenerate the myocardium. ${ }^{7}$ Studies demonstrate this approach improves or sustains cardiac function after implantation onto damaged rat heart muscle. ${ }^{7}$ ECTs, derived from human cells, also, improve cardiac function in a rat cardiac MI model. ${ }^{8}$ However, this model has many barriers before clinical translation: determination of optimal tissue size, immune system rejection, obvious demonstration of ECT electrical coupling to the host heart, limitations on cell availability, and immature phenotype. 
Regarding phenotype, ECTs have many immature characteristics. ${ }^{9}$ The maturity of ECTs surpasses 2D culture; however, no ECT method produces a fully mature construct. ${ }^{9}$ The constructs produce inadequate force, the tissues possess underdeveloped contractile machinery, and the contractile units also spontaneously beat. ${ }^{9}$ In contrast, native cardiac tissue produces much greater force, the mature muscle has an abundance of contractile proteins, and the cells elongate and align. ${ }^{9}$ To improve cardiac maturity, researchers use bioreactors, that mimic the in vivo environment, to promote differentiation. ${ }^{10}$ Many methods replicate these in vivo signals, such as, mechanical stretch, medium perfusion, and electrical stimulation. ${ }^{9}$ Nutrient delivery, in tissue engineering, is usually limited to a tissue area that can be reached by diffusion; perfusion increases nutrient and oxygen transport, allowing construction of uniform and thick tissue constructs. ${ }^{10}$ Mechanical stretch manipulates integrin binding which activates pathways related to hypertrophy: MAPK/ERK1/2 and PI3K. ${ }^{11}$ Changes in stiffness manipulate tyrosine kinase activation and GTPase activity which activates pathways such as Rho/ROCK to differentiate cells. ${ }^{11}$

Electrical stimulation promotes differentiation into cardiomyocytes by replicating endogenous electric fields present during cardiac development. ${ }^{12}$ These endogenous electric fields influence cellular behavior, proliferation, and migration. ${ }^{13}$ Absence of these endogenous electric fields in development generate severe defects such as malformed body parts. ${ }^{13}$ Even though endogenous electric fields are known to generate these effects, the mechanism behind electrically-induced maturation still requires explanation. $\cdot{ }^{14}$ Using a neonatal ventricular rat myocyte, NVRM, culture model, this electrical stimulation mechanism has been profusely studied..$^{15,16,17}$ 
An initial study, by McDonough and Glembotski, electrically stimulated NVRMs by direct stimulation with an electric field. ${ }^{13,15}$ They placed charged electrodes in the cell media on opposite sides of the cell culture, establishing an electric field between the cells. ${ }^{15}$ They showed long term electrical field stimulation of NVRMs induces a hypertrophic-like response in the cells. ${ }^{15}$ The cardiomyocytes enlarged, they organized their myofibrils, and expression levels of MLC-2 (myosin light chain) and ANF (atrial natriuretic factor) increased in response to electric stimulation. ${ }^{15}$ This study, and following studies by the same group, focused on the mechanism of this electricallyinduced hypertrophic response. ${ }^{15}$

McDonough and Glembotski used drug treatments which clarified the mechanism of electrical stimulation. ${ }^{15}$ Phenylephrine (PE) stimulated phosphatidylinositol (PI) hydrolysis and caused an increase in ANF; however, electrical stimulation did not stimulate PI hydrolysis, suggesting electrical stimulation increases ANF through a pathway independent of protein kinase $C .{ }^{15}$ Moreover, chelerythrine, a PKC antagonist, did not inhibit pacing-induced ANF secretion, further supporting the aforementioned statement. ${ }^{16}$ McDonough and Glembotski also demonstrated that, unlike with forskolin stimulation, electrical stimulation did not increase cAMP formation, suggesting an ANF signal transduction pathway independent of protein kinase $A \cdot{ }^{15}$ On the contrary, nifedipine and W7, a calmodulin inhibitor, blocked ANF secretion, suggesting dependence of the response to electrical stimulation on calcium influx and the calcium-calmodulin pathway. ${ }^{15}$ 
Based on these results, McDonough and Glembotski suggested a partial mechanism for electrical stimulation. ${ }^{15}$ Calcium influx, as a result of an action potential, induces calcium-induced calcium release from the sarcoplasmic reticulum; the increase in cytoplasmic calcium activates CaMK, and the activated CaMK phosphorylates CREB which allows CREB to bind to CRE-like sequences in the $5^{\prime}$ region of the ANF gene, stimulating the hormone's transcription. ${ }^{15}$ McDonough et al. further elucidated the mechanism for electrical stimulation by demonstrating pacing-induced activation of MAPK family member, JNK. ${ }^{17}$ Calcium transients could activate CaMKs that induce JNK activity. ${ }^{17}$ Then, JNK phosphorylation of c-jun could precede transcription of ANF. ${ }^{17}$

The hypertrophic response, observed by McDonough et al., also involves mitochondrial proliferation and maturation. ${ }^{18}$ Xia et al. demonstrated electrical stimulation increases cytochrome oxidase mRNA, cytochrome oxidase enzyme activity, and number of mitochondria. ${ }^{18}$ Malonyl-CoA, a metabolic inhibitor of the CPT-1 enzyme, more effectively inhibited electrically stimulated cells. ${ }^{18}$ This enhancement of inhibition suggested a shift from the liver isoform of CPT-1 to the muscle isoform of the enzyme. ${ }^{18}$ In fact, they showed electrical stimulation also increased expression of the muscle isoform of the CPT-1 protein. ${ }^{18}$

The mechanism underlying these cellular changes involves the transcription of certain genes in the nucleus. ${ }^{19,20}$ Expression of c-Fos/c-jun/junB, AP-1 proteins, and NRF1 preceded cytochrome c mRNA transcription. ${ }^{19,20}$ Supplementing this observation, mutations of the NRF-1 binding site and a possible AP-1 binding site, CRE, on the Cyt C gene diminished Cyt c transcriptional response to electrical stimulation. ${ }^{19}$ These results 
suggested NRF-1 and AP-1 contribute to the initiation of mitochondrial proliferation in response to electrical stimulation. ${ }^{19}$ In a later study, NRF-1 and c-jun binding to the Cyt c promoter was clearly demonstrated, illustrating their direct involvement in electricallyinduced mitochondrial maturation. ${ }^{20}$ Along with induction of mitochondrial proliferation and activity, electrical stimulation also influences metabolic genes. ${ }^{21}$

Xia et al. showed electrical stimulation upregulates the muscle-specific Adss1 gene, a gene encoding a purine metabolism enzyme, and the stimulation represses the nonmuscle gene, Adss $2 .{ }^{21}$ Furthermore, they demonstrated this upregulation involves the calcineurin/NFAT pathway. ${ }^{21}$ Calcineurin dephosphorylates NFAT, allowing NFAT to translocate to the nucleus to transcribe the Adss1 gene. ${ }^{21}$ Cyclosporine $A$, an inhibitor of calcineurin, inhibited electrical induction of the Adss1 gene, suggesting the involvement of calcineurin. ${ }^{21}$ Also, cells containing mutant NFAT binding sites could not enhance transcription of the Adss1 gene in response to electrical stimulation. ${ }^{21}$ Also, mutant GATA sites inhibited pacing-induced Adss1 gene transcription. ${ }^{21}$ Taken together, these results suggest electrical pacing activates the calcineurin/NFAT3 and GATA4 pathways. ${ }^{21}$ Simultaneously, as electrical stimulation manipulates metabolism, autocrine growth factor signaling may also play a role in the electrically-induced hypertrophic response. ${ }^{22}$

Kaye et al. illustrated paced adult ventricular rat cells released more basic fibroblast growth factor, and electrical stimulation increased protein content and cell size. ${ }^{22}$ This electrically-induced hypertrophic response could be inhibited by an anti-FGF antibody. ${ }^{22}$ Additionally, unstimulated cells exposed to basic fibroblast growth factor exhibited a hypertrophic response. ${ }^{22}$ These results suggest electrically-induced 
mechanical contractions alter membrane permeability, allowing release of basic fibroblast growth factor. ${ }^{22}$ This result reveals the interplay between mechanical contractions and electrical stimulation during the hypertrophic response resulting from pacing. ${ }^{22}$ Cooperative interaction may also exist between electrical stimulation and reactive oxygen species (ROS) production to induce hypertrophy. ${ }^{14}$

Electrical stimulation is known to produce ROS, and, although large amounts of ROS damage cells, small amounts of ROS can activate pathways related to growth and differentiation. ${ }^{14}$ Serena et al. electrically stimulated human embryoid bodies (EBs) derived from human embryonic stem cells; the EBs differentiated into cardiomyocytes while, also, producing ROS. ${ }^{14}$ This result suggests ROS production in response to electrical stimulation may induce cardiomyocyte differentiation. ${ }^{14}$ Another study, by Chan et al., also cited ROS as a second messenger that can activate signaling cascades related to growth and differentiation. ${ }^{23}$

Calcium cycling in the cytosol has been highlighted as essential to the growth and differentiation response; however, calcium may also contribute to electrically-induced hypertrophy by other means. ${ }^{15,24}$ Electrical stimulation of human atrial cardiac progenitor cells induced greater calcium cycling in the nucleus than in the cytosol. ${ }^{24}$ Therefore, electrically-induced calcium cycling may cause activation of calcium dependent proteins and transcription factors involved in differentiation. ${ }^{24}$ Calcium also influenced alignment of cardiomyocytes. ${ }^{24}$ Calcium-free media caused cardiac progenitor cells to align less parallel to the electric field lines. ${ }^{24}$ In contrast, calcium-rich media induced parallel alignment to the electric field lines. ${ }^{24}$ 
Calcium signaling is complemented by VEGF production in the mechanistic response to electrical pacing, and this VEGF production can induce angiogenesis in immature cardiac cells. ${ }^{25}$ Rackauskas et al., utilizing subthreshold high-frequency electrical field stimulation on NVRMs, induced secretion and protein expression of VEGF. ${ }^{25}$ Furthermore, VEGF secretions into the cell media promoted proliferation of human coronary artery endothelial cells, promoting angiogenesis. ${ }^{25}$ This result suggests electrical stimulation can induce angiogenesis through VEGF production. ${ }^{25}$

Because electricity induces cardiac maturity, many clinical cell therapy and tissue engineering research methods apply electrical stimulation paradigms. Skeletal progenitor cells, used for cell therapy, respond to electrical pacing similarly to cardiac cell types. ${ }^{26,4}$ C2C12 muscle cells cultured and electrically stimulated on Ultrafoam collagen sponges deposited less collagen 1 , correlating with less scar formation. ${ }^{26}$ The electrically stimulated $\mathrm{C} 2 \mathrm{C} 12$ myotubes contracted effectively, and the stimulation improved ultrastructure such as sarcomere organization. ${ }^{26}$ Likewise, Pedrotty et. al demonstrated electrical stimulation induces cell proliferation of skeletal myoblasts on PGA scaffolds, but the cells failed to differentiate into cardiac cells. ${ }^{27}$ While these results relate to cells from a skeletal origin, electrical stimulation can also manipulate differentiation of stem cells, used in cardiac regeneration, such as mesenchymal and adipose derived stem cells. ${ }^{4,28,29,30}$

Electrically stimulated mesenchymal stem cells seeded with carbon nanotubes elongated, aligned perpendicular to the electric field, and increased expression of cardiac markers. ${ }^{29}$ Human adipose derived stem cells, in response to DC electric stimulation 
elongated and aligned perpendicular to the electric field, possibly, to minimize voltage across the cell. ${ }^{30}$ Thrombomodulin, VEGF, and FGF upregulated in response to stimulation. ${ }^{30}$ Connexin-43 upregulated after the first two hours of stimulation, but, subsequently, the junction downregulated after four hours of stimulation. ${ }^{30}$ Therefore, connexin disassembly and alignment simultaneously occur in these cells. ${ }^{30} \mathrm{In}$ a following study, Pavesi et al. showed similar increased expression of connexin-43 from biphasic electrical stimulation of mouse adipose-derived stem cells. ${ }^{31}$ As suggested earlier, electrical stimulation does not rely upon the absence of a cardiac phenotype; cardiac cells destined to be a cardiac cell can be further driven towards a mature cardiac phenotype by electrical stimulation.

NVRMs, electrically stimulated and seeded on ultrafoam collagen sponges, gained improvements in contractility, expression of cardiac proteins, cardiac differentiation, and ultrastructural organization. ${ }^{32}$ Amplitude of contraction, excitation threshold (minimum voltage at set pacing frequency), and maximum capture rate (highest pacing frequency) improved in stimulated constructs. ${ }^{32}$ Electrical stimulation elevated levels of troponin, creatine kinase-MM, and myosin heavy chain. ${ }^{32}$ Stimulated constructs displayed mature $\mathrm{H}, \mathrm{I}$, and A bands, and myofibers aligned along electric field lines. ${ }^{32}$ However, cellular maturation depended on onset of electrical stimulation: initiation of electrical stimulation on day 3 improved cellular phenotype better than electrical stimulation initiation on day 1 or $5 .^{32}$ Electrical stimulation alone does not induce fully developed cardiac constructs; electrical stimulation of cardiac progenitor cells combined with other differentiation cues can improve the recapitulation of the native myocardium. ${ }^{9,33}$ 
Heidi Au et al. electrically stimulated fibroblasts and neonatal rat ventricular cardiomyocytes on microabraded and non-abraded surfaces to study the combined and separate effects of these cues. ${ }^{33}$ Micoabrasions replicated the topographical cues in the native myocardium that guide orientation and elongation of cells. ${ }^{33}$ Combinations of these cues improved alignment and elongation compared with just electrical stimulation alone. ${ }^{33}$ Electrical stimulation and microabrasion influenced cell elongation similarly, but microabrasion had greater influence over cell orientation. ${ }^{33}$ Cell morphology and the contractile apparatus improved the most by combination of abrasions and electrical stimulation compared with electrical stimulation alone. ${ }^{33}$ Along with topographical cues, the native heart also contains dense capillary network that minimizes nutrient and oxygen transport. $^{10}$

To mimic this situation in vitro, Maidhof et al. simultaneously perfused and stimulated NVRMs seeded on porous PGS scaffolds. ${ }^{10}$ Medium perfusion through porous PGS scaffolds enhanced nutrient transport, and the pores protected cells from hydrodynamic shear, a morphology-altering force. ${ }^{10}$ The bioreactor allowed free contraction of the construct to retain differentiation induced by mechanical stretch. ${ }^{10}$ Perfusion and electrical stimulation of porous PGS scaffolds containing NVRMs improved excitation threshold (ET), maximum capture rate (MCR), and amplitude of contraction compared to electrical stimulation alone. ${ }^{10}$ Also, this combination maintained DNA content, increased creatine kinase expression, and increased troponin content as compared to electrical stimulation alone. ${ }^{10}$ As cited previously, mechanical stretch and 
electrical stimulation also combine to improve cardiac maturity. The following study more explicitly demonstrates this tendency.

Wang et al. studied the effects of mechanical stretch and electrical stimulation of acellular porcine myocardial scaffolds seeded with mesenchymal stem cells. ${ }^{28}$ Synergistic effects of mechanical stretch and electrical stimulation improved recellularization, tissue remodeling, and cardiac differentation. ${ }^{28}$ Enhanced properties included improvement of cell alignment, induction of nonlinear anisotropic behavior, and formation of cell junctions. ${ }^{28}$ These constructs expressed myosin heavy chain, alpha actinin, and cardiac troponin T. ${ }^{28}$ Interestingly, alignment only occurred in the presence of mechanical stimulation; whereas, electric fields alone could not reorient the cells. ${ }^{28}$ Physical stressors play an important role in cardiac induction, but growth factors can also induce differentiation.

Park et al. investigated the effects of IGF-1 exposure on electrically stimulated cardiac constructs. ${ }^{34}$ Cardiac construct viability, differentiation, and functional properties benefit from IGF-1 exposure. ${ }^{34}$ This combination increased connexin-43 expression, sarcomere development, and 2D projection length, a contractility indicator compared with electrical stimulation alone. ${ }^{34}$ Electrical stimulation induced cell alignment along electric field lines regardless of IGF-1 activity. ${ }^{34}$ The group surmised a mechanism for these results; the anti-apoptotic effects and pro-differentiation effects of IGF-1 synergized with electrical stimulation's induction of contractile protein assembly. ${ }^{34}$ Combinations of growth factors and electrical stimulation induce substantial cell 
differentiation; however, interest lies in improvement of electrical stimulation rather than combination of ES (electrical stimulation) with other protocols. ${ }^{29,35}$

Nanowires, deposited in the cardiomyocyte cell culture, also, synergistically improve electrical stimulation. ${ }^{35}$ Richards et al. electrically stimulated human induced pluripotent stem cell derived cardiac spheroids containing conductive silicon nanowires. ${ }^{35}$ The stimulation of the conductive silicon wires reduced spontaneous beating similar to with electrical stimulation alone, decreasing the risk for arrhythmogenicity complications after transplantation. ${ }^{35}$ They suggested reduction of HCN4 mRNA, RNA encoding a pacemaking channel, caused a decrease in spontaneous beating. ${ }^{35}$ Silicon wire stimulation significantly increased expression of connexin-43 and a mechanical junction, $\mathrm{N}$-cadherin in comparison with electrical stimulation without nanowires. ${ }^{35}$ High levels of mechanical junctions may improve cellular integration after transplantation. ${ }^{35}$ Stimulated/nanowired spheroids expressed enough sarcomeric proteins to generate sarcomeric banding; whereas, electrical stimulation alone produced less mature sarcomeres. $^{35}$ These spheroids, in comparison with electrical stimulation alone, also, handled calcium more effectively through increased expression of the ryanodine receptor. ${ }^{35}$ Beta-MHC and cardiac troponin increased expression in wired/stimulated constructs, in comparison to electrical stimulation alone, consistent with in vivo cardiomyocyte maturation. ${ }^{35}$ Wired and stimulated spheroids increased expression of myosin-light chain 2 on the gene and protein level in comparison with electrical stimulation alone, suggesting a shift towards a ventricular phenotype. ${ }^{35}$ 
In a similar study, Ahadian et al., using dielectrophoresis, aligned carbon nanotubes in methacryloyl hydrogel scaffolds. ${ }^{36}$ Electrical stimulation of the scaffolds, containing mouse derived cardiac EBs, improved cardiac differentiation compared with electrical stimulation alone. ${ }^{36}$ Scaffolds with aligned carbon nanotubes, following stimulation, compared with electrical stimulation alone, increased troponin T expression, and the excitation upregulated the cardiac genes: Tnnt2, Nkx2-5, and Actc1. ${ }^{36}$ In contrast to the previous study, spontaneous beating of the cardiac EBs cultured with carbon nanotubes increased in response to electrical stimulation compared with electrical stimulation alone. ${ }^{36}$ Manipulation of stimulation parameters can also optimize electrical response rather exogenous addition of conductive materials.

Tandon et al. found electrode length must be twice the electrode spacing for optimal electrical pacing, and electric field strength must be $8 \mathrm{~V} / \mathrm{cm}$ or lower to retain construct viability. ${ }^{37}$ In a following study, Tandon et al. showed placement of constructs within a $4 \mathrm{~mm}$ distance from the midway point between electrodes exposes cells to a linear electric field. ${ }^{38}$ Also, carbon electrodes stimulated tissues most effectively. ${ }^{38}$ These electrodes retained stimulus, and the electrodes injected and recovered the most charge, a potential toxicity to cells. ${ }^{38}$ Also, carbon induced maturation of functional properties, MCR and ET, most effectively. ${ }^{38}$ By comparing functional properties of tissues paced with different stimulation protocols, Tandon et al. identified optimal stimulation parameters for electric field strength, frequency, and pulse duration: $3 \mathrm{~V} / \mathrm{cm}, 3 \mathrm{~Hz}, 2 \mathrm{~ms} .{ }^{38}$

Another stimulation parameter for optimization of the waveform involves the phasic nature of the electrical propagation as mentioned earlier. ${ }^{31}$ Electrical stimulation 
can be either monophasic or biphasic. ${ }^{38}$ Biphasic electrical stimulation requires less energy, is more efficacious, and the reduces charge buildup that causes cellular damage. ${ }^{38,39}$ Chiu et al. used biphasic electrical stimulation on PEG discs seeded with embryoid body-derived mouse endothelial cells, NVRM cardiomyocytes, and NVRM fibroblasts. ${ }^{39}$ Biphasic stimulation more effectively increased cell density, formation of contractile organoids, connexin-43 expression, and proliferation of fibroblasts in comparison with monophasic stimulation. ${ }^{39}$ Furthermore, biphasic stimulation may have induced growth factor secretion, leading to fibroblast growth. ${ }^{39}$ Pietronave et al. used biphasic stimulation on c-kit/Sca-1-expressing human cardiac progenitor cells (hCPCs) ${ }^{40}$ Biphasic stimulation, in comparison with monophasic stimulation, more effectively upregulated transcription factors for cardiac development after three days of stimulation: MEF2D, GATA4, and Nkx2.5. ${ }^{40}$ Furthermore, connexin- 43 , troponin $T$, and a calcium channel (Cav2.1) similarly upregulated in response to biphasic stimulation in comparison with monophasic stimulation. ${ }^{40}$ Finally, biphasic stimulation, in comparison with monophasic stimulation, also, increased localization of connexin-43 to the cell membrane, and stimulated cells tended orient perpendicular to the electric field..$^{40}$ While these studies provide a basis for electrical stimulation, most important to the research presented here is previous work concerning electrical stimulation of ECT constructs.

ECT constructs under electrical stimulation, also, differentiate into more mature tissues. Lasher et al. chronically stimulated rat ECTs, inducing improved myocyte volume fraction, alignment, and enhanced connexin- 43 distribution. ${ }^{41}$ Control unstimulated ECTs possessed sparse connexin- 43 connections, indicating the immature electrophysiology in 
these constructs; whereas, more mature postnatal myocardium expressed higher connexin-43 proteins. ${ }^{41}$ The improved connexin-43 distribution shows electrical stimulation drives maturity to a postnatal phenotype. Excitation threshold and maximum capture improved in electrically stimulated constructs. ${ }^{41}$ Hirt et al. electrically stimulated human induced pluripotent stem cell-derived ECT constructs. ${ }^{42}$ Stimulated human ECTs produced higher force, and stimulated ECT orientation and histological maturity improved compared to unstimulated constructs. ${ }^{42}$ Moreover, a stimulation protocol that combined periods of $2 \mathrm{~Hz}$ and $1.5 \mathrm{~Hz}$ stimulation improved cell maturity better than a $.5 \mathrm{~Hz}$ stimulation protocol. ${ }^{42}$ Nunes et al. constructed and electrically stimulated ECTs by seeding human stem cell derived cardiomyocytes in collagen gels around a conductive surgical suture. ${ }^{43}$ Electrical stimulation of these biowires enhanced ultrastructure, electrophysiological properties (MCR and ET), and electrically stimulated biowires possessed increased potassium channel (KCNJ2) mRNA compared to age-matched embryoid bodies. ${ }^{43}$ Surprisingly, a ramp-up protocol from $1 \mathrm{~Hz}$ to $6 \mathrm{~Hz}$ produced the greatest maturation even though stimulation surpassed physiological frequency. ${ }^{43}$ Godier-Furnemont et al. subjected NVRM derived circular ECTs to chronic electrical stimulation, inducing a positive-force frequency relationship in the ECT ${ }^{44} \mathrm{~A}$ positive-force frequency relationship is a force increase as frequency progresses to higher stimulation rates. ${ }^{44}$ The positive-force frequency relationship seems to depend on mature intracellular calcium stores which allow rapid calcium cycling. ${ }^{44}$ Correlating with this underlying mechanism, these ECTs possessed matured T-tubules, SERCA2a protein expression, and RYR2 protein, providing a basis for the positive force-frequency 
relationship. ${ }^{44}$ Also, an enhancement in frequency-dependent acceleration of ECT relaxation and calcium sensitivity accompanied the positive force-frequency relationship. ${ }^{44}$

We tested electrical stimulation on our ECTs, incorporating human induced pluripotent stem cell derived cardiomyocytes (h-iPSC-CMs), but we have failed to observe an increase in cardiomyocyte maturity. As an explanation of this result, electrical stimulation does seem to be somewhat h-iPSC cell line dependent. ${ }^{12}$ Besides these poor results, this stimulation paradigm has other hindrances. Electrical stimulation can be invasive, requiring insertion of electrodes into the ECT. ${ }^{43}$ During electrical stimulation, Faradaic reactions occur which release toxic substances into cell media, limiting electrical pulses to under $1 \mathrm{~ms}$, and electrical stimulation produces $\mathrm{pH}$ alterations. ${ }^{45,46,47}$ Electrical stimulation also has low spatiotemporal resolution because the excitation is not cellspecific, and the stimulation has no innate negative feedback control. 45,48

An alternative, and possibly better, strategy to electrical stimulation is optogenetics. ${ }^{45}$ Optogenetics involves heterologous expression and light stimulation of channelrhodopsin light sensitive ion channels, derived from alga, to produce action potentials. ${ }^{49}$ After heterologous expression of channelrhodopsin, absorption of blue light causes an all-trans retinal chromophore, in the channel, to isomerize to 13 -cis retinal. ${ }^{49}$ This isomerization opens the ion channel, allowing sodium ions into the cell which produces this action potential. ${ }^{49}$ Darkness causes the chromophore to assume its natural trans conformation which, subsequently, closes the channel. ${ }^{49}$ Boyden et al. first heterologously expressed channelrhodopsins in mammalian cells, and, since this initial 
study, many research endeavors in neuroscience and cardiology, use channelrhodopsins for controlled action potential generation in cells. ${ }^{49,50,51}$ The light sensitive ion channels used for interrogation include chimeric and mutant channelrhodopsin variants with improved properties: higher conductance (ChR2/H134R), improved kinetics (ChETA), and lower light desensitization (ChIEF). ${ }^{52}$ Rhodopsin proteins also exist that inhibit action potential initiation: halorhodopsin, an inward chloride pump, and archaerhodopsin, an outward proton pump. ${ }^{53}$

Many advantages exist for this stimulation paradigm in comparison to electrical stimulation. Because the cell membrane expresses these ion channels, this stimulation paradigm can occur at a cell-specific level, increasing spatiotemporal resolution for more precise investigation of electrophysiology. ${ }^{45,48}$ Introduction of channelrhodopsins eliminate the need for electrodes; therefore, light stimulation is less invasive. ${ }^{43}$ Also, toxic byproduct release is uncommon with channelrhodopsin excitation, and, because the stimulation directly opens channels with a reversal potential, light stimulation has innate negative feedback. ${ }^{45}$ Application of optogenetics to cardiac research is relatively new; however, the studies, thus far, demonstrate the advantages of this technique. ${ }^{54,55,56,57,58}$ Cardiac optogenetic investigation initially started when Bruegmann et al. produced a channelrhodopsin expressing embryonic mouse stem cell line. ${ }^{46}$ Cardiomyocytes, differentiated from this stem cell line, contracted in response to blue light. ${ }^{46}$ Calcium imaging demonstrated light pulses induced calcium transients in the cardiomyocytes, and longer light pulses increased release of calcium. ${ }^{46}$ Light-induced stimulation generated propagation through a syncytium of cardiomyocytes plated on 
multielectrode arrays, and point stimulation allowed propagation initiation at different points in the syncytium. ${ }^{46}$

Cardiomyocytes can also couple to optically responsive noncardiac cells, and the cardiomyocytes contract in response to optical excitation of the coupled cells. ${ }^{51}$ Jia et al. transfected human embryonic kidney (HEK) cells with channelrhodopsin..$^{51}$ Using patch clamp and calcium imaging, light stimulation elicited action potential currents in the HEK cells, and these currents propagated to co-cultured neonatal rat cardiomyocytes and adult canine cardiomyocytes. ${ }^{51}$ Cabenoxolone, a gap junction uncoupler, eliminated propagation of these light-induced currents to co-cultured cardiomyocytes, providing evidence for current initiation from light sensitive HEK cells and propagation of these currents through gap junctions to the co-cultured cardiomyocytes. ${ }^{51}$ Propagation occurred in cell pairs and, also, through two-dimensional layers of cells. ${ }^{51}$ Action potentials, calcium transients, wave propagation, and conduction velocities were similar to electrical stimulation. ${ }^{51}$ Furthermore, light stimulus caused, not only action potential propagation, but, also contraction of the cardiomyocytes. ${ }^{51}$ They showed low irradiance needed for excitation, indicating the possible energy efficiency of this approach compared to electrical stimulation. ${ }^{51}$

Cardiac optogenetics extended to cardiomyocytes derived from human embryonic stem cells when Abilez et al. transduced human embryonic stem cells with a channelrhodopsin lentiviral vector; these stem cells, after differentiation into cardiomyocytes, electrically responded and contracted to blue light stimulation. ${ }^{49}$ Abilez also provided evidence for rhodopsin functionality in h-iPSC-CMs. ${ }^{47} \mathrm{H}$-iPSC-CMs, 
expressing channelrhodopsin and halorhodopsin, responded to light, increasing or decreasing contraction rate. ${ }^{47}$ Along with acute stimulation, Abilez also provided a LED array for chronical stimulation and subsequent differentiation of optically responsive hiPSC-CMs. ${ }^{59}$

Optogenetic functionality can also be extended to 3D tissues. Sakar et al. expressed channelrhodopsin in $\mathrm{C} 2 \mathrm{C} 12$ myotubes, and skeletal microtissues formed from these myotubes contracted in response to optical excitation. ${ }^{60}$ In a similar study, Chan et al. generated neonatal rat ECTs seeded with channelrhodopsin-expressing HEK293T cells. ${ }^{61}$ These ECTs contracted in response to OP, demonstrating the feasibility of optogenetics in ECTs constructs. ${ }^{61}$

Optogenetics could also be used as tool for cardioversion and pacemaking. ${ }^{62}$ Atrial fibrillation is often treated with drug therapy or electrical cardioversion despite the proarrhythmic and painful tissue-damaging effects of these therapies, respectively. ${ }^{54}$ Bingen et al. showed, by expressing a channelrhodopsin in neonatal rat atrial monolayers, that reentrant spiral waves, a symptom of atrial fibrillation, can be terminated with optogenetic excitation. ${ }^{54}$ Optogenetic stimulation increased functional core size of spiral waves, resulting in phase singularity drift that caused collisions which terminated the spiral waves. ${ }^{54}$ Essentially, this paradigm provides a painless shock-free means of cardioversion. ${ }^{54}$ Supplementing this study, Burton et al. demonstrated direction reversal of spiral wave rotation, further exhibiting the high spatiotemporal resolution of this optogenetic technique. ${ }^{63}$ 
Drawbacks exist with pacemakers, as well, and these hindrances can be improved through use of optogenetics. Pacemakers need constant battery replacements, the electronic components do not replicate the native pacemaker, and pacing leads do not reach certain anatomical regions. ${ }^{62}$ The biological nature of an optogenetic pacemaker could remedy these issues. ${ }^{62}$ Nussinovitch et al. theorized an implantable optogenetic cell graft could act as a biological pacemaker. ${ }^{62}$ Progressing towards this goal, Nussinovitch et al. coupled optogenetic fibroblasts with human and rat cardiomyocytes. ${ }^{62}$ Light stimulation of the fibroblasts generated contraction in the cardiomyocytes, synchronized non-coupled cardiomyocytes, and activated multiple specific sites in the culture. ${ }^{62}$ These results provide preliminary evidence for efficacy of this optogenetic approach in conduction abnormality treatment. ${ }^{62}$ This approach could also potentially treat tachyarrhythmias as ArchT, an inhibitory rhodopsin, suppressed contractions in the fibroblast-cardiomyocyte co-cultures. ${ }^{62}$

Following initial successful in vivo optogenetic experiments by Bruegmann et al. and Arrenberg et al., Nussinovitch and Gepstein used an adeno-associated viral vector containing channelrhodopsin to infect rat hearts. ${ }^{46,56,64}$ Optical excitation of channelrhodopsin paced the whole heart, and channelrhodopsin activation, at multiple sites, resynchronized the ventricles. ${ }^{64}$ Recently, Nyns et al. provided support for a feasible optogenetic pacemaking and defibrillation therapy. ${ }^{55}$ The group demonstrated optogenetic termination of rat ventricular tachyarrhythmias in a Langendorff-apparatus, possibly through prolongation of action potential duration. ${ }^{55}$ 
In addition, in vivo OP can answer basic science questions concerning the propagation and termination of arrythmias. ${ }^{65}$ Zaglia et al. used optogenetics to reveal the minimum cell number required for ectopic beats, the arrhythmogenic potential of different heart areas, and the role of different cell types. ${ }^{65}$ For example, ectopic beats only required OP of 1,000 to 2,000 cardiomyocytes, but only 100 Purkinje fibers, under $\mathrm{OP}$, generated ectopic beats, suggesting a higher arrhythmogenic potential of conducting cardiomyocytes. ${ }^{65}$

Optogenetics is also conducive to high-throughput drug testing unlike traditional electrophysiological approaches. ${ }^{57}$ An all-optical approach, using voltage-sensitive and calcium-sensitive fluorescent dyes as readouts in combination with optogenetic actuation, does not require tedious contact with the cell, and light stimulation can test many cultures in parallel, further contributing to rapid readout times. ${ }^{57}$ Rapid readout times result in more efficient identification of toxic drug candidates. ${ }^{57}$ In this regard, Klimas et al. developed OptoDyCE, an all-optical automated high-throughput drug testing system. ${ }^{57}$ This system tests drugs by combining optogenetic actuation and optical sensing of readouts such as voltage. ${ }^{57}$ OptoDyCE is amenable to 96 -well formats and 384 -well formats, allowing many parallel experiments. ${ }^{57}$ In a similar study, Dempsey et al. developed Cardiac Optopatch: a high-throughput drug testing system combining simultaneous OP, voltage imaging, and calcium imaging. ${ }^{58}$ Optopatch efficiently detects drug-induced effects on action potential morphology and calcium transients which could improve drug testing protocols based on hERG blockade. ${ }^{58}$ 
To supplement these studies and extend optogenetics to the field of chronic ECT stimulation, in my thesis related study, we expressed ChIEF in h-iPSC-CM ECTs using an adeno-associated virus packaged with this rhodopsin. We investigated the ion channel's functionality in the constructs by patch clamp. After chronic stimulation for 7 days, we assessed the ECTs for maturity, using force testing, histology, RT-qPCR and alignment testing. 
MATERIALS AND METHODS

Differentiation of Human iPS cells into Cardiac Cells

We used two h-iPS cell lines: 201B6 and 863B3. Kyoto University shipped these cell lines to University of Louisville as a component of a Collaborative Research Agreement. Retroviral introduction of Oct3/4, Sox2, KIf4, c-Myc generated 201B6. Introduction of non-integrating episomal plasmids encoding L-Myc, LIN28, Glis1, Sox2, KIf4, and Oct3/4 generated 863B3. We cultured h-iPSC cell lines on matrigel (growth factor reduced, 1:60 dilution; BD Biosciences, San Jose, CA) in human basic fibroblast growth factor (hbFGF; WAKO, Osaka, Japan), $4 \mathrm{ng} / \mathrm{ml}$, augmented mouse embryonic fibroblast conditioned medium (MEF-CM). We subcultured small clusters (every 4-6 days) in CTK solution [0.25\% Trypsin, $1 \mathrm{mmol} / \mathrm{L} \mathrm{CaCl}_{2}$ in phosphate buffered saline (PBS), $20 \%$ knockout serum replacement (KSR), and $0.1 \%$ collagenase IV]. To induce cardiac differentiation, we removed cells from culture plates by incubating the cells in Versene (0.48 mmol/L EDTA solution; Life Technologies, Carlsbad, CA) for 3 to 7 minutes. At a density of 1,000 cells $/ \mathrm{mm}^{2}$, we plated these cells onto matrigel-coated plates in MEF-CM augmented with $4 \mathrm{ng} / \mathrm{ml} \mathrm{hbFGF}$ for a few days before differentiation. A day before induction, we covered the cells with matrigel (1:60 dilution). We differentiated cells into cardiac cells using the following steps. On differentiation day 0 , we replaced MEF-CM with RPMI+B27 medium (RPMI1640; Life Technologies 2 mmol/L L-glutamine; 
LifeTechnologies, 1x B27 supplement without insulin: Life Technologies) augmented with Activin A (R\&D, Minneapolis, MN) and Wnt3a (R\&D) both at a concentration of $100 \mathrm{ng} / \mathrm{ml}$ for one day. The following day, differentiation day 1 , for 2 or 4 days with no media change, we exposed the cells to $10 \mathrm{ng} / \mathrm{ml}$ human bone morphogenetic protein 4 (BMP4; R\&D) and $10 \mathrm{ng} / \mathrm{ml}$ hbFGF. We replaced the culture medium on day 5 with RPMI+B27 augmented with $50 \mathrm{ng} / \mathrm{ml}$ of VEGF 165 (Miltenyi, Bergisch Gladbach, Germany) to differentiate cells into a mixture of cardiomyocytes and endothelial cells ( $\mathrm{CM}+\mathrm{EC}$ protocol). We changed the medium every 48 hours. On day 11 to day 13 of induction, the cells began to beat. In accordance with the cardiomyocyte and mural cell induction protocol $(\mathrm{CM}+\mathrm{MC})$, we cultured the cells in RPMI+B27 augmented with insulin (Life Technologies) and $100 \mathrm{ng} / \mathrm{ml}$ of Dkk1 (R\&D) starting on day 5. We cultured the cells in this medium until day 7 while changing the media every other day. To generate only mural cells (MC protocol), we replaced the media with RPMI+10\% FBS medium [RPMI1640, $2 \mathrm{mmol} / \mathrm{L}$ L-glutamine, $10 \%$ fetal bovine serum (FBS)] on differentiation day 3 . We changed this culture media every day, as well.

Lineage Analysis

On day 15 of differentiation, we added Accumax (Innovative Cell Technologies, San Diego, CA) to dissociate cells from cell culture plates. We stained the cells with the following surface markers: anti-VCAM1 conjugated with allophyco-cyanin (APC, BioLegend), anti-PDGFRß conjugated with phycoerythrin (PE, BD, Franklin Lakes), anti-VEcadherin conjugated with fluorescein isothiocyanate (FITC, BD), and anti-TRA-1-60 conjugated with FITC (BD). We stained the cells with LIVE/DEAD Aqua dead cell staining 
kit (Invitrogen). Finally, we stained the cells with anti-cardiac isoform of Troponin T (cTnT, Thermo-Fisher) tagged with Alexa-488 (Invitrogen). We stained the cells in PBS with 5\% FBS for cell surface markers. We stained cells fixed with $4 \%$ paraformaldehyde (PFA) for intracellular proteins. We analyzed the stained cells with a BD LSRII Flow Cytometer and DIVA software (BD).

Engineering of ChIEF-Expressing Engineered Cardiac Tissue

After lineage analysis, we used a varying distribution of differentiated cells (cardiomyocytes, endothelial cells, and mural cells) for engineering of control and ChIEFexpressing ECTs. We combined three million cells with matrix factors to construct one linear tissue. First, we suspended the three million cells in 83 ul of culture medium (Modified Dulbecco's Essential Medium, Invitrogen) containing 20\% FBS (Invitrogen) in a $15 \mathrm{ml}$ tube. Then, we neutralized acid-soluble rat-tail collagen type-1 solution (Sigma) $(\mathrm{pH}=3$ in $\mathrm{HCl})$ with alkali buffer $(0.2 \mathrm{M} \mathrm{NaHCO} 3,0.2 \mathrm{M}$ HEPES, $0.1 \mathrm{M} \mathrm{NaOH})$ on ice in a separate PCR tube. We added Matrigel to the collagen solution at $15 \%$ of the total volume. We mixed the cell suspension and matrix solution at a collagen concentration of $0.67 \mathrm{mg} / \mathrm{ml}$. The final volume of the cell/matrix mixture is approximately $200 \mathrm{ul}$. We transfected the cell/matrix mixture with an AAV1/2-CAG-oChIEF-tdTomato virus (a gift from the Guido lab, originally produced by VectorBioLabs, Malvern, PA). We used the virus $\left(2 \times 10^{12} \mathrm{VG} / \mathrm{ml}\right)$ at an $\mathrm{MOI}$ of $500\left(1.5 \times 10^{9}\right.$ viral particles per 3 million cells in each ECT). We poured the 200ul of the cell/virus/matrix mixture into a vacuum-suctioned trough (20mm length $\times 2 \mathrm{~mm}$ width) composed of a Flexcell Tissue Train collagen type-1 coated silicone membrane (TissueTrain; Flexcell International, Hillsborough, FL). We 
controlled vacuum-suction with a FX-5000TT system (Flexcell International). The vacuumsuctioned trough had anchors on either end to hold and shape the tissue into a linear format. The mixture was vacuum suctioned in an incubator for $120 \mathrm{~min}\left(37^{\circ} \mathrm{C}, 5 \% \mathrm{CO}_{2}\right)$. After gel compaction induced by vacuum suction, we added pre-culture medium [PM; alpha minimum essential medium ( $\alpha$ MEM; Life Technologies) augmented with 10\% FBS, $100 \mathrm{U} / \mathrm{ml}$ Penicillin-Streptomyocin (Life Technologies), and 5×10-5 M 2-mercaptoethanol (Sigma)]. We maintained ECTs at $37^{\circ} \mathrm{C}$ and $5 \% \mathrm{CO} 2$ to culture day 14 (D14), changing media every other day. We additionally performed prolonged culture to D28 for a set of non-transfected, non-paced ECTs.

Chronic Light Stimulation

We clearly defined the chronic light stimulation protocol in Figure 1B. Between D7 and D14 of ECT culture, we performed chronic optical pacing (C-OP) of transfected ECTs with a custom-built LED system. The system consisted of 470-nm wavelength blue LEDs (Luxeon, Alberta, Canada) driven by BuckPuck DC LED drivers (LEDsupply, Randolph, VT). We mounted the LEDs on a high-alpha heat sink (Luxeon) for thermal management. We controlled the pulse width and frequency of the LEDs using an Arduino Uno microcontroller (Italy). We programmed the Arduino with free Arduino IDE software. Using this LED system, we adjusted the pacing rate every day to at least $0.5 \mathrm{~Hz}$ below the maximum capture rate. We kept the pulse width at $5 \mathrm{~ms}$. We assessed maximum capture rate by observing pacing through a microscope camera (model MU1000, AmScope, Irvine, CA). Maximum capture rate was the highest pacing frequency before initiation of 
asynchronous contractions. We used custom video tracking software if assessment of maximum capture rate was difficult.

Force Testing

Before force measurement, we removed the linear tissue from the FlexCell culture plate. We placed the tissue in cold Tyrode's solution $\left(25^{\circ} \mathrm{C}\right)$ in a perfusion chamber containing $95 \% \mathrm{O}_{2}$ and $5 \% \mathrm{CO}_{2}$. The Tyrode's solution was composed of (in mmol/L) 119.8 $\mathrm{NaCl}, 5.4 \mathrm{KCl}, 2.5 \mathrm{CaCl}_{2}, 1.05 \mathrm{MgCl}_{2}, 22.6 \mathrm{NaHCO}_{3}, 0.42 \mathrm{NaH}_{2} \mathrm{PO}_{4}, 0.05 \mathrm{Na}_{2} \mathrm{EDTA}, 0.28$ ascorbic acid, 5.0 glucose, and 30 2,3-butanedione monoxime (BDM). Using 10-0 suture, we tied the tissue to a stainless steel bar, in the perfusion chamber, connected to a force transducer (model 403A; Aurora Scientific, Ontario, Canada). We attached the opposite end to a stainless steel bar, high-speed length controller (model 322C, Aurora Scientific), connected to a micromanipulator. We, then, filled the perfusion chamber with warmed Tyrodes's solution $\left(37^{\circ}, 2 \mathrm{ml}\right.$ volume) containing no BDM. We preconditioned the construct for 20 minutes at slack length with no stimulation. Using the micromanipulator, we moved the length controller to stretch the construct to maximum length (Lmax). At maximum length, we performed force measurements with a series of optical and electrical stimulation protocols. We optically stimulated the transfected tissue at $5 \mathrm{~ms}$ pulse widths with an Arduino powered blue LED, and we electrically stimulated the constructs with electrodes in the perfusion chamber. During both optical and electrical stimulation protocols, we increased pacing frequency by $0.5 \mathrm{~Hz}$ from a $2 \mathrm{~Hz}$ baseline until we obtained maximum capture rate. We measured excitation threshold by pacing the construct at $2 \mathrm{~Hz}$ and decreasing the voltage until the construct could no longer capture. 
Using custom FFT analysis software on the force traces from optical and electrical stimulation protocols, we retrieved active stress $\left(\mathrm{mN} / \mathrm{mm}^{2}\right)$, intrinsic beat rate, maximum capture rate $(\mathrm{Hz})$, normalized force-frequency, beat variation, relaxation time to $50 \%$ (RT50), and systolic energy at $3 \mathrm{~Hz}$.

Patch Clamp

Patch clamp recordings were performed similarly to previously described protocols. ${ }^{66}$ At D14, we removed the ECT from the FlexCell culture plate, and placed the tissue in a recording chamber continuously perfused with the same BDM-infused Tyrode's solution $\left(2.5 \mathrm{ml} / \mathrm{min}, 95 \% \mathrm{O}_{2} / 5 \% \mathrm{CO}_{2}\right)$ used in force testing. We maintained the solution at $37^{\circ} \mathrm{C}$. For differential interference contrast imaging of ChIEF expression, we used a $10 \mathrm{x}$ or 60x water-immersion objective (Olympus) and CCD camera on an upright microscope (Olympus BX51WI) with a filter (Chroma 49005) for tdTomato (tdT) expression. Then, we pulled borosilicate glass capillaries using a vertical puller (Narishige), and they were filled with the following solution: $117 \mathrm{mM} \mathrm{K}$-gluconate, $13.0 \mathrm{mM} \mathrm{KCl}, 1 \mathrm{MgCl}_{2}, 0.07 \mathrm{CaCl}_{2}, 0.1$ EGTA, 10 HEPES, 2 Na-ATP, and 0.4 Na-GTP. We maintained internal solution osmolality and internal solution $\mathrm{pH}$ at $290 \mathrm{mOsm}$ and 7.3, respectively. We patched the filled borosilicate capillary onto a single cell in the ECT. We used a Multiclamp 700B amplifier (Molecular Devices) for whole cell recordings.

Light from a blue light emitting diode (Prizmatix UHP 460) reflected into the $4 x$ or 60x objective. This light optically activated the ChIEF-expressing ECT. The LED light for the $4 \mathrm{x}$ and the $60 \mathrm{x}$ objective was 2.2 or $.45 \mathrm{~mm}$, respectively. We controlled pulse duration and frequency through software. A Multiclamp 700B amplifier (Molecular Devices) 
recorded from the single cell during optical activation of the whole ECT. Filtered data was analyzed with pClamp 10 software (Molecular Devices).

Histology \& Alignment

After ECT fixation in 4\% PFA for 30 minutes at room temperature, we rinsed the ECTs in 1xPBS. We then cut the ECTs in half along the short-axis cross section. We washed the halves in 1\% Triton-X-100/PBS for 1 hour at RT. We blocked with 1\% Triton-X-100/PBS $+10 \%$ FBS for 1 hour at RT. We incubated the tissues with a primary antibody for cTnT (mouse, Ms-295, Thermo Fisher Scientific, Cambridge, US) at a ratio of 1:400 in 1\% Triton$\mathrm{X}-100 / \mathrm{PBS}+10 \% \mathrm{FBS}+0.02 \%$ sodium azide overnight at $4^{\circ} \mathrm{C}$. After this overnight incubation, we washed with 1\% Triton X-100/PBS+10\% FBS for 1 hour and 1\% Triton X100/PBS for three 10 minute intervals. We used a donkey anti-mouse IgG secondary antibody (molecular probes, Oregon, USA) conjugated to Alexa 488 the following day. We incubated the ECTs with this antibody, after another washing with $1 \%$ Triton-X-100/PBS+ $10 \% \mathrm{FBS}+0.2 \%$ sodium azide $+5 \%$ donkey serum, overnight at $4^{\circ} \mathrm{C}$. Following staining with DAPI (R37606, molecular probes, Oregon, USA) for 30 minutes at room temperature, we incubated ECTs overnight in $100 \%$ glycerol followed by $75 \%$ glycerol for 2 hours, and then with a clearing solution (53\% benzyl alcohol, 45\% glycerol, and 2\% DABCO) for 2 hours. We then changed to a new clearing solution overnight for incubation in the dark. We stored processed samples in clearing solution at $4^{\circ} \mathrm{C}$ for further imaging.

We performed IF staining also on 5- $\mu$ m sections. To generate these sections, after fixation in 4\% PFA for 30 minutes at room temperature, the tissues were processed to paraffin blocks and cut to $5-\mu \mathrm{M}$ sections, using a microtome. We blocked the sections 
with 0.1 M Glycine + 0.5\% Triton X-100 in PBS. We used a primary antibody cTnT (Ms-295, Thermo Fisher Scientific, Cambridge, US) at a ratio of 1:400, and we used a Living Colors DsRed Polyclonal Antibody (Cat\# 632495, Clontech, Takara Bio USA, Inc.) at a ratio of 1:300. We incubated sections with these antibodies in $1 \%$ BSA overnight at $4^{\circ} \mathrm{C}$. The following day, we incubated sections with Alexa Flour 488 donkey anti-mouse IgG and Alexa Flour 594 donkey anti-rabbit IgG secondary antibodies (molecular probes, Oregon, USA) in 5\% donkey serum for 1.5 hours at room temperature. We mounted slides with ProLong ${ }^{\circledR}$ Gold Antifade Reagent with DAPI (molecular probles, Oregon, USA).

We used a Nikon ECLIPSE Ti Confocal System (Nikon, Tokyo, Japan) attached to a Nikon Ti-E inverted microscope platform. We captured whole mount tissue images for alignment analyses with a 10X 0.3 NA objective at $1024 \times 1024$ pixel density and a 3- $\mu$ m z-interval up to 150 steps (447 $\mu \mathrm{m})$. We captured images for cardiomyocyte-density and ChIEF-tdT transfection efficiency with a 40x 1.30 NA objective at $1024 \times 1024$ pixel density and $1-\mu \mathrm{m}$ z-interval up to 5 steps, covering $2 \times 3$ fields. We captured images using Nikon NIS Elements AR software (Nikon), and we saved the 12-bit raw files for further processing.

Using 3D confocal stacks of whole-mount cTnT immunostained samples, we quantified alignment of cardiomyocytes in ECT bundles. Analysis of $n=9$ and $n=10$ images was completed for the control ECT group and the transfected paced ECT group, respectively. The mean images size was $1.3 \times 1.7 \times 0.45 \mathrm{~mm}$. After attenuation correction and low pass Gaussian filtering (standard deviation of $3 \mu \mathrm{m}$, ImageJ, NIH, Bethesda, MD), we merged the cTnT, tdT, and DAPI fields into a single grayscale image and manually 
segmented the whole ECT bundle with seg3D (Salt Lake City, UT). A coordinate system was formed by extracting the centerline (medial axis) from the distance transform of the segmented image. From the intensity gradient of the cTnT image, we calculated local cardiomyocyte orientations and quantified alignment based on spherical statistics. Alignment quantification was performed in Matlab (Mathworks, Natick, MA).

Real-Time Quantitative Polymerase Chain Reaction

We performed the following steps for both control and transfected paced constructs. We cut the constructs from the collagen type-1 coated silicone membrane 6well plate (TissueTrain; Flexcell International, Hillsborough, FL). We added 2 to 4 ECT samples into a $5 \mathrm{ml}$ tube filled with $1 \mathrm{ml}$ of Trizol buffer (Life Technologies, Carlsbad, CA). We homogenized the constructs in the Trizol buffer with an Omnitip Tissue homogenizer (Cat. No.: 6615-7273, USA Scientific, Ocala, FL) for 40 to 60 seconds at full speed on ice. We incubated the homogenates at room temperature for 5 minutes. We added $200 \mu \mathrm{l}$ of chloroform (Sigma, St. Louis, MO) to the samples, we vortexed the samples, and we incubated them at room temperature for another 5 minutes. We centrifuged samples for 20 minutes at $10,000 \mathrm{rpm}$ at $4^{\circ} \mathrm{C}$. After collecting the supernatant to a new tube, we added $450 \mu \mathrm{l}$ to $550 \mu \mathrm{l}$ of $70 \%$ ethanol. We applied $700 \mu \mathrm{l}$ of the sample to an RNeasy mini column (Qiagen, Valencia, CA; Cat. No.: 74104) and centrifuged the column for 15 seconds at 10,000 rpm, discarding the flow through. We applied 700 ul of RW1 (Qiagen, Valencia, CA; Cat. No.: 74104) onto the RNeasy column to a new $2 \mathrm{ml}$ collection tube, and we centrifuged the column for 15 seconds at 10,000 rpm, discarding the flow through. We, then, applied $500 \mu \mathrm{l}$ of RPE buffer (Qiagen, Valencia, CA; Cat. No.: 74104) onto the 
column, and we centrifuged for 15 seconds at 10,000 rpm, discarding the flow through. This step was repeated. We placed the column in a new $2 \mathrm{ml}$ collection tube, and we centrifuged for 1 minute at full speed to dry the column. We then transferred the column to a new $1.5 \mathrm{ml}$ RNase and DNase free tube (Qiagen, Valencia, CA; Cat. No.: 74104). We pipetted $25 \mu \mathrm{l}$ of RNase-free water directly onto the membrane, and we centrifuged the tube for 1 minute at 10,000 rpm. We measured RNA concentration and purity using a NanoDrop 2000 (Thermo Fisher Scientific, Waltham, MA). We stored RNA samples at $80 \circ \mathrm{C}$

We used a reverse transcription kit (Invitrogen, Carlsbad, CA; Cat. No: 11754-050). For a single reaction, we combined the following components in a tube on ice: $4 \mu$ of $5 x$ VILO Reaction Mix, $2 \mu \mathrm{l}$ of 10x SuperScript Enzyme, $\leq 2.5 \mu \mathrm{g}$ of RNA, and enough diethylpyrocarbonate-treated water (DEPC-treated water) to make the total volume 20 $\mu \mathrm{l}$. We gently mixed the contents. We incubated the tubes at $25^{\circ} \mathrm{C}$ for $10 \mathrm{mins}, 42^{\circ} \mathrm{C}$ for 60 mins, and we terminated the reaction at $85^{\circ} \mathrm{C}$ for 5 minutes. We added $80 \mu$ l of DNase free water to the sample, and we aliquoted the samples to $8 \mu \mathrm{l} /$ tube. We stored cDNA samples at $-80^{\circ} \mathrm{C}$ or $-20^{\circ} \mathrm{C}$.

We performed quantitative polymerase chain reaction (qPCR) with a Taqman ${ }^{\circledR}$ Gene Expression Master Mix and an ABI 7900HT (Applied Biosystems, Foster City, CA). For each qPCR experiment, we pooled 3 ECTs. We performed 4 qPCR experiments for both control and paced ECTs. We added $225 \mu \mathrm{l}$ of $\mathrm{ddH}_{2} \mathrm{O}$ to $25 \mu \mathrm{l}$ of cDNA $(250 \mu \mathrm{l})$ on ice. We did this for each cDNA group used. We added $70 \mu$ of Taqman Master mix and $7 \mu$ of primer to a tub on ice. We did this for each primer used. We added $11 \mu \mathrm{l}$ of each 
Taqman/primer mixture to 3 wells of a 96-well plate (Corning Incorporated, Corning, NY) for each analyzed group on ice. To those triplicate wells, we added $9 \mu$ of cDNA to each well on ice. We completed qPCR using software on the ABI 7900HT (Applied Biosystems, Foster City, CA).

Statistical Analyses

We statistically compared D14 control $(n=13)$ and transfected paced tissues $(n=15)$. We also statistically compared transfected paced ECTs $(n=15)$ to a prolonged culture D28 tissues $(n=2)$. To establish a comparison between two groups, we used a ttest at a significance level of $p<0.05$. 


\section{RESULTS}

ChIEF transfection of h-iPSC derived cardiomyocytes within ECTs and chronic optical pacing

Following initial pilot experiments using several AAV constructs and transfection doses, we transfected day $14 \mathrm{~h}$-iPSC derived cardiac cells with an AAV1/2-CAG-ChIEFtdTomato virus at an $\mathrm{MOI}$ of 500 by adding virus directly to the cell/matrix mixture at the time of ECT formation (Figure 1A). ChIEF transfected h-iPSC-derived ECTs displayed spontaneous beating starting around D3 of our standard 14 day in vitro culture, similar to non-transfected control ECTs. Starting at D7 of culture, we began C-OP with a custom LED system (Figure $1 \mathrm{~A})$. We recorded intrinsic beat rate, maximal OP rate, and then set C-OP rate at greater than intrinsic but less than maximal OP rate (Figure 1B). Before initiation of C-OP experiments, we confirmed OP depolarization and action potential generation of cardiomyocyte within transfected ECTs using pulsed blue light stimulation and patch clamp recording (Figure $2 \mathrm{~A})$.

Force testing of ChIEF h-iPSC ECTs confirm functional maturation

The following text describes force testing parameters and the definitions of these readouts can be found in Figure S2. ChIEF-transfected ECTs were harvested at D14 and control h-iPSC derived ECTs at D14 or D28 to quantify functional maturation. Optical 
pacing during in vitro force testing confirmed pacing capture (Figure 2B). Electrical field pacing was used for all quantitative force-length and force-frequency data collection and we noted rate dependent changes in both passive and active stress (Figure 2E) typical of immature myocardium. The first indicator that C-OP increased ECT functional maturation is the observation that C-OP ECTs displayed a higher electrical field paced MCR than D14 control ECTs $(5.37 \pm 0.69$ versus $4.35 \pm 0.59 \mathrm{~Hz}, \mathrm{p}<0.001$, Figure $2 \mathrm{C})$ though the MCR for C-OP D14 ECTs was less than the MCR for D28 non-transfected ECTs $(5.37 \pm 0.69$ $\mathrm{Hz}$ versus $6.50 \pm 0.71 \mathrm{~Hz}, \mathrm{p}<0.05$, Figure $2 \mathrm{C}$ ). Intrinsic beat rate increased in chronically optically paced ECTS inconsistent with maturation compared with both D14 and D28, but only significantly with D14 control constructs $(1.46 \pm 0.34 \mathrm{~Hz}$ D14 and $1.80 \pm 0.28 \mathrm{~Hz}$ D28 versus $2.37 \pm 0.43 \mathrm{~Hz}$ TP500, $\mathrm{p}<0.01$, Figure $2 \mathrm{C}$ ). Consistent with cardiomyocyte maturation, RT50 measured at $3.0 \mathrm{~Hz}$ decreased in chronically optically paced ECTs compared to D14 controls ( $88.00 \pm 9.60$ versus $104.23 \pm 6.66 \mathrm{~ms}, p<0.001$, Figure $2 \mathrm{D}$ ) and further decreased by D28 (65.60 \pm 2.12 versus $88.00 \pm 9.60 \mathrm{~ms}, \mathrm{p}<0.05$, Figure $2 \mathrm{D})$. The second indicator that chronically OP increased ECT functional maturation is the observation that chronically optically paced ECTs displayed a less negative stressfrequency relationship than D14 control ECTs, though D28 ECTs displayed a less negative stress-frequency relationship (Figure 2E). Consistent with C-OP induced maturation in calcium cycling, we noted reduced beat-to beat amplitude variation in active stress ( $\left.\sigma_{b b}\right)$ at higher frequencies in C-OP ECTs versus D14 ECTs (Figure 2F). Of note, beat-to beat amplitude variation in active stress ( $\left.\sigma_{b b}\right)$ was negligible by D28 (Figure $2 \mathrm{~F}$ ). Active stress $\left(\sigma_{\mathrm{A}}\right)$ was similar between D14 and C-OP ECTs $\left(0.72 \pm 0.39\right.$ versus $0.52 \pm 0.27 \mathrm{mN} / \mathrm{mm}^{2}$, 
$p=0.14$, Figure $2 G)$. Active stress trended lower for D28 ECTs $(0.17 \pm 0.11$ versus $0.72 \pm 0.39$ $\mathrm{mN} / \mathrm{mm}^{2}, \mathrm{p}=0.08$, Figure $2 \mathrm{G}$ ) consistent with reduced cardiomyocyte survival following prolonged ECT in vitro culture. Systolic energy, a normalized contractile efficiency index calculated at $3 \mathrm{~Hz}$ significantly decreased in C-OP ECTs compared to D14 controls $\left(0.47 \pm 0.03\right.$ versus $0.54 \pm 0.02 \mathrm{~J} / \mathrm{m}^{3}-\mathrm{s}, \mathrm{p}<.001$, Figure $\left.2 \mathrm{H}\right)$. As expected, systolic energy was lower in the more functionally mature D28 ECTs compared to C-OP ECTs $(0.41 \pm 0.01$ versus $0.47 \pm 0.03 \mathrm{~J} / \mathrm{m}^{3}-\mathrm{s}, \mathrm{p}<.05$, Figure $2 \mathrm{H}$ ).

Chronic optical pacing does not accelerate structural maturation

ChIEF-transfected and control h-iPSC ECTs were also harvested after D14 culture to quantify the impact of C-OP on structural maturation. Whole h-iPSC ECTs (Figure 3A) were placed into 2D paraffin blocks for serial section processing (Figure 3B and $3 \mathrm{C}$ ) or stained as whole mounts for 3D confocal imaging (Figure 3D, 3G, and 3I). ChIEF proteins predominantly co-located with CTnT positive cells consistent with preferential cardiomyocyte transfection (Figure 3C). Control and chronically optically paced ECTS displayed similar cardiomyocyte percentages ( $50 \pm 13$ vs $48 \pm 13$, Figure $3 E$ ). Transfected ECTs possessed $20 \pm 6 \%$ ChIEF-transfected cardiomyocytes (Figure 3E, inset). In contrast to the functional maturation noted after 7 days of C-OP, cardiomyocyte alignment was similar between control and chronically optically paced ECTs (alignment concentration ( $\mathrm{k}$ ) $=3.76 \pm 1.11$ vs $3.42 \pm 1.33$, Figure $3 F$ ). The $3 \mathrm{D}$ distribution and alignment of local cardiomyocyte orientations were also similar between control (Figure 3G) and chronically optically paced (Figure 3I) ECTs consistent with the similar active stresses noted for these D14 h-iPSC ECT groups (Figure 2G). 
Chronic optical pacing and extended culture is associated with upregulation of mature cardiac RNA markers

We performed RNA analysis on multiple biological replicates of D14 control and transfected paced constructs (Figure 4). MRNA for KCNJ2, SERCA2A, and cTnT, common markers for maturity, did not upregulate for transfected paced tissues (Figure 4). The ratio of MLC2v and MLC2a, an indicator of ventricular phenotype development, insignificantly increased (Figure 4A). KCND3 increased 2-fold in transfected paced constructs, suggesting this channel partially underlies electrophysiological improvements (Figure 4B).

A single biological replicate of RT-qPCR was completed on RNA isolated from a D28 linear h-iPSC ECT (Figure 5) as has been previously completed for D28 large format h-iPSC ECTs. Common structural and electrophysiological genes were upregulated in D28 constructs: RYR2, SERCA2A, Cacna1D, SCN4A, KCND3 (Figure 5). The types of genes increased reflect the superior electrophysiological properties of extended ECT culture. SERCA2A and RYR2 could underlie the superiority of D28 constructs. 


\section{DISCUSSION}

Our findings show the ChIEF AAV virus preferentially transfected h-iPSC derived cardiomyocytes that then expressed ChIEF and enabled acute and chronic ECT OP conditioning. Histological immunostaining of 2D paraffin sections showed co-localization of cTnT and ChIEF expression (Figure 3C). This co-localization provides evidence that OP directly perturbed the cardiomyocyte membrane to produce action potentials and contractions. Furthermore, this result supports a previous report by Abilez et al. showing efficient expression of channelrhodopsins in cardiomyocytes derived from human induced pluripotent stem cells. ${ }^{47}$

Alignment analysis of 3D whole mount images showed OP did not affect orientation of cells (Figure 3F). This result suggests OP does not affect pathways related to migration like endogenous electric fields. ${ }^{13}$ In vitro electrical stimulation, unlike OP, correlates with cellular alignment in some cases. ${ }^{24,29,30,32,33,34,40,41,42}$ However, the effect of electrical stimulation on alignment is rather unclear. For example, some groups observed perpendicular reorientation to the electric field; whereas, other studies observed parallel alignment to the electric field. ${ }^{24,29,30,32,34,40}$ Radisic et al. suggested parallel alignment underlies decreased excitation threshold because parallel alignment optimizes use of the voltage gradient, and, in a contradictory statement, Tandon et al. 
supposed perpendicular alignment minimizes exposure to high voltage. ${ }^{30,32}$ Furthermore, Heidi Au et al. demonstrated topographical cues influence alignment more than electrical stimulation, and Wang et al. observed alignment only with the presence of mechanical stimulation. ${ }^{28,33}$ Also, electrically induced alignment also seems to depend on the initiation time of the electrical stimulation. ${ }^{32}$ For example, initiation of electrical stimulation on day 5 failed to improve contractile apparatus organization. ${ }^{32}$ The different cell lines used in these studies could explain the differing effects of electrical stimulation on alignment. As mentioned before, electrical stimulation seems to be cell line dependent. ${ }^{12}$ On the contrary, these results could suggest electrical stimulation could possess little influence over alignment because of the inconsistent results, and the alignment is actually the product of other cell signals such as topography. These previous observations imply explanations for our own study.

Chronic OP ECT alignment may depend on cell line, or OP, like electrical stimulation, could possibly have little effect on alignment compared to other signals. ${ }^{28,33}$ Also, here, we initiated C-OP on D7; C-OP may depend on time of initiation like electrical stimulation. ${ }^{32}$ Finally, the frequencies, in this study, differed from previous ECT electrical stimulation studies demonstrating alignment induction. Frequencies generally stayed around $3 \mathrm{~Hz}$ for the full week of pacing; whereas, Nunes et al. and Hirt et al. showed most success with a $6 \mathrm{~Hz}$ and $2 \mathrm{~Hz} / 1.5 \mathrm{~Hz}$ protocol, respectively. ${ }^{42,43}$

Cardiomyocyte percentage was similar between control and transfected ECTs, and the virus transfected approximately $20 \%$ of the total cardiomyocyte population (Figure 3E). Similar cardiomyocyte percentages demonstrate the safety of AAV transfection. In 
fact, we partially chose an AAV vector because the safety of these viruses; therefore, this result reinforced the validity of our decision to utilize this viral type. ${ }^{67,68}$ Also, the effective transfection illustrates the feasibility of heterologous expression in these ECTs, and the result reinforces our decision to use an AAV1. We based this decision on this serotype and virus possessing an outstanding capacity to transfect h-iPSC-CMs. ${ }^{68}$ Finally, the number of cardiomyocytes transfected suggest OP only requires a subset of ChIEF-expressing cells. OP may excite a subset of cells which induce action potential propagation, through cellcell connections such as gap junctions to other cells.

Simultaneous patch clamp and OP demonstrated action potential generation in response to light stimulation (Figure $2 \mathrm{~A}$ ). Efficient cardiomyocyte stimulation requires action potential generation; therefore, action potential generation is important to validate in response to OP. ${ }^{69}$ However, action potential generation must be coupled to contraction, called excitation contraction coupling in native tissue. ${ }^{69}$ Therefore, we showed OP could produce force-generating contractions (Figure 2B). During force testing, OP frequencies could reach up to $5 \mathrm{~Hz}$, as confirmed by pacing at this frequency. Optical MCR was comparable to the average maximum capture electrical stimulation, $5.37 \mathrm{~Hz}$. Therefore, the limits of optical and electrical stimulation may be similar.

Chronic OP was successfully performed for seven days (Figure 1B). Successful COP supports the decision to use ChIEF, and this success further demonstrates the validity of using an AAV vector. We chose ChIEF because the channel does not desensitize with chronic light stimulation, and, partially, we chose an AAV because of the vector's persistent transgene expression. ${ }^{52,68}$ Because of the success of C-OP, these two properties 
proved to be useful in C-OP. Success shows the light channel did not desensitize and remained stably expressed throughout the seven-day period. Also, successful chronic pacing demonstrates the utility our custom-built LED system loosely based on the LED model system developed by Abilez..$^{59}$

Chronic OP significantly increased both MCR and intrinsic beat rate from D14 controls (Figure 2C). However, MCR of extended D28 ECTs was significantly higher than transfected paced constructs. MCR is an indicator of improvement in electrical properties because a higher MCR coincides with increased connexin43 expression and potassium channel expression. ${ }^{43,70}$ MCR increased previously due to extended culture, providing a basis for the result of extended culture in the present study, and further associating extended culture with maturation. ${ }^{70}$ The result of C-OP is similar to chronic electrical pacing studies, demonstrating MCR improvements. ${ }^{10,38,43}$ Most importantly, this improvement in MCR was comparable to a previous ECT chronic electrical stimulation study. Nunes et al. showed chronic electrical stimulation increased MCR by approximately $1 \mathrm{~Hz} .{ }^{43}$ We show C-OP also increased MCR of ECTs by approximately $1 \mathrm{~Hz}$. However, we improved MCR without invading the tissue, and we did not immobilize the tissue, maintaining differentiation by mechanical stretch unlike Nunes. ${ }^{11,43}$

With regard to the change in intrinsic beat rate, C-OP did not induce a decrease in spontaneous beating indicative of maturation (Figure 2C). ${ }^{9}$ Our result differed with a previous study concerning electrical stimulation of human induced pluripotent stem cell derived cardiac spheroids. That study, by Richards et. al, showed a decrease in spontaneous beating rate, possibly resulting from a decrease HCN4, a pacemaking 
channel. ${ }^{35}$ However, our result agreed with a study that used electrical stimulation of mouse derived cardiac EBs. ${ }^{36}$ This study, by Ahadian et al., demonstrated electrical pacing increased spontaneous beating rate. ${ }^{36}$ Possibly, electrical stimulation affects intrinsic beat rate of various cell types differently, and the same result exists with chronic light pacing. OP may induce a consistent upregulation in HCN4 channels unlike normal electrophysiological maturation.

Along with an improvement in MCR, C-OP significantly decreased beat-to-beat variation at all frequencies and relaxation time compared to D14 controls (Figure 2D, 2F). Similar to MCR, D28 constructs possessed lower beat-to-beat variation and significantly different relaxation time than transfected paced constructs. This result was consistent with a previous study associating extended ECT culture with improved relaxation time. ${ }^{70}$ Like MCR, improved hysteresis and relaxation time correlate with increased connexin43 expression and upregulation of potassium channel expression. ${ }^{70}$ Godier-Furnemont et al. also showed an improvement in relaxation time due to electrical stimulation of ECT constructs similar to OP. ${ }^{44}$ Mathematical modeling demonstrates hysteresis occurs due to out-of-phase action potentials and calcium cycling. ${ }^{71}$ Thus, we also propose the decreased hysteresis indicates maturation of excitation-contraction coupling.

Chronic OP ECTs maintained active stress, as a function of increasing pacing frequency, better than D14 control constructs (Figure 2E). D28 constructs maintained active stress better than transfected paced constructs at most frequencies, but D28 constructs were only significantly different at $4.5 \mathrm{~Hz}$ and $5 \mathrm{~Hz}$. These results further associate extended ECT culture with maturation as observed in a previous study. ${ }^{70}$ Better 
maintenance of active stress allows coupling to higher pacing rates in vivo without sacrificing force production. ${ }^{8}$ The underlying mechanism for improved maintenance of active stress production correlates with increased expression and development of calcium handling structures. ${ }^{44}$ Calcium handling stores and proteins allow improved stress preservation because these proteins cause quicker release and reuptake of calcium, the ion involved in force production. ${ }^{44}$ However, unlike Godier-Furnemont et al. electrical stimulation study, C-OP did not generate a positive force frequency relationship. ${ }^{44}$ Moreover, C-OP did not induce a positive-force frequency relationship as seen in D28 constructs in a previous study. ${ }^{70}$

We did note that absolute active stress trended lower in chronically OP constructs compared to D14 controls but higher than D28 constructs (Figure 2G). Despite increased functional maturation defined by ECT electrical properties, active stress trended downward with extended culture, perhaps due to limited nutrient diffusion or cell death ${ }^{10}$ Possibly, optogenetic stimulation, ChIEF expression, or AAV transfection decreased active stress through some toxicity. Optogenetic stimulation may generate hydrodynamic shear, a morphology-altering force. ${ }^{10}$ This result is in contrast to chronic electrical stimulation. Chronic electrical stimulation of ECT constructs increased active force by 1.5 times. ${ }^{42}$ However, our result may differ due to differences in the stimulation protocols. For example, chronic electrical stimulation involved pacing at $2 \mathrm{~Hz} / 1.5 \mathrm{~Hz}$; whereas, our protocol usually paced tissues at around $3 \mathrm{~Hz} .{ }^{42}$ Lower pacing rates may be more conducive to stimulating sarcomere maturation and increased active stress. Possibly, 
survival in ECT is cell-line dependent and another cell line would possess improved survival after C-OP or extended culture.

Transfected paced constructs used significantly less systolic energy during contraction at $3 \mathrm{~Hz}$ pacing compared to D14 control constructs (Figure $2 \mathrm{H}$ ). However, D28 constructs used significantly less systolic energy than transfected paced constructs (Figure $2 \mathrm{H}$ ). Systolic energy use was significantly different as indicated by decreased stress-time integral values. The stress-time integral relates to energy consumption during contraction. ${ }^{72}$ Increased expression of mitochondrial genes and mitochondria number could underlie this result as observed in electrical pacing of neonatal rat cardiomyocytes. ${ }^{18}$ Possibly, CPT-1 expression could be transitioning to the muscle isoform of the CPT-1 enzyme. ${ }^{18}$

RT-qPCR analysis suggests KCND3, the gene encoding the Kv4.3 channel, the channel responsible for the transient outward $I_{\text {to, }}$ at least partially underlies the electrophysiological maturation induced by C-OP (Figure 4B). ${ }^{70,73}$ This channel provides a partial mechanism for electrophysiological maturation as the mRNA was the only gene significantly upregulated gene in transfected paced constructs (Figure 4B). KCND3 increases with extended culture, signifying its role in maturity. ${ }^{70}$ Furthermore, increased expression of Kv4.3 could improve hysteresis and MCR because the channel decreases repolarization time; overexpression of Kv4.3 in isolated guinea pig myocytes decreased membrane potential during the plateau phase and shortened action potential duration. ${ }^{74}$ Surprisingly, RNA analysis did not reveal significant upregulation of calcium ion channels or SERCA2A to explain active stress preservation (Figure 4A). Possibly, Western blot 
analysis would reveal differences at the protein level, or the degree of protein phosphorylation and activation, as observed previously with electrical pacing. ${ }^{44}$ This RNA result was consistent with a previous ECT electrical stimulation study that showed no upregulation of SERCA2A. ${ }^{42}$ The one biological replicate of D28 control constructs suggests far greater expression of mature cardiac proteins in comparison with transfected paced constructs (Figure 5). D28 constructs possessed greater expression of the following mRNA compared to transfected paced constructs: MLC2v/MLC2a, RYR2, SERCA2, Cacna1D, SCN4A, and KCND3. The larger number of increased mRNA, especially RYR2 and SERCA2, could underlie the significantly improved electrophysiological characteristics of D28 constructs (Figure 5A).

As stated previously, ECTs possess an immature phenotype, limiting their clinical translation as a cardiovascular regeneration therapy or viable drug testing platform.9,57 Bioreactors that mimic the in vivo environment induce differentiation and maturation. ${ }^{10}$ The in vivo signals replicated include mechanical stretch, perfusion, and electrical stimulation. ${ }^{9}$ However, no ECT differentiates into a fully mature construct. ${ }^{9}$ Also, electrical stimulation can be invasive, produces toxic Faradaic reactions, and has low spatiotemporal resolution. ${ }^{43,45,46,47,48}$ Because ECTs still retain immaturity and chronic electrical stimulation has hindrances, the cardiac tissue engineering field needs alternative, less toxic, conditioning protocols that produce a level of maturity comparable to electrical stimulation. We present here a novel, alternative, chronic, less toxic, action potential inducing, stimulation protocol for cardiac tissue engineering, using expression of light sensitive ion channels. After extensive review, this is the first successful attempt 
at C-OP of engineered ECTs consisting of h-iPSC cardiac cells. Furthermore, we provide evidence for the possibility of functional maturation, utilizing this technique. Also, the light stimulation protocol developed is economically feasible, only requiring easily obtainable components, and the Arduino hardware and IDE is user-friendly.

The results of our current study may have alternate interpretations. The electrophysiological maturation observed could be due to indirect effects of C-OP. For example, light stimulation, by indirectly causing increased mechanical stretch or perfusion, could induce electrophysiological maturity. ${ }^{9,10}$ Possibly, C-OP causes secretion of growth factors, such as VEGF and bFGF, into the supernatant similar to previous electrical stimulation studies. ${ }^{22,25}$ Furthermore, our cell population consists of a mixture of cardiomyocytes, endothelial cells, and mural cells. We could not control the ratios between these cell types because differentiation produces a different mixture of cells for every experiment. We used the same hIPS cell stock for each differentiation protocol; however, many ECTs were made from a different differentiated cell lot. A different cell population for every experiment may influence results because a previous study, by our lab, shows ratio of cell types influences electrophysiological properties. ${ }^{8}$ Presence of certain cells can activate pathways related to tissue maturation. ${ }^{8}$ Also, stem cell derived cardiomyocytes co-cultured with endothelial cells beat more synchronously, suggesting cell population variability could have an effect on efficacy of C-OP. ${ }^{75}$

Limitations also exist in the present study. AAV transfection or ChIEF expression may generate an immune rejection after ECT implantation; however, primate brains express channelrhodopsins for long periods of time with persistence and lack of toxic side 
effects, suggesting long-term in vivo implantation may be possible. ${ }^{51}$ Furthermore, we do not know whether we used the most efficient virus or channelrhodopsin variant. Also, we did not fully optimize the viral dose; a smaller viral dose used could reduce toxicity and improve transfection efficiency. Also, we did not account for transfected endothelial cells and mural cells which could affect dose. Channelrhodopsins can express in vascular endothelial cells, and these endothelial cell populations can respond to light with shifts in membrane potential. ${ }^{76}$ Therefore, transfected EC cells could have an effect on maturity induced by C-OP. Further optimization of the light pacing protocol could improve ECT maturity, and optimization could improve active stress measurements. For example, the length of $\mathrm{C}-\mathrm{OP}$ and the time of initiation for $\mathrm{C}-\mathrm{OP}$ requires optimization. The length, frequency, and pulse width for pacing was arbitrarily chosen based on successful OP capture. Experimental groups in the study could be improved. The number of $d 28$ groups was small compared to other groups, and we did not use a group to directly assess the toxicity of the light itself. Furthermore, an underlying mechanism for the functional data requires further explanation.

To improve these limitations, a comparison between different variants of channelrhodopsins and viruses could elucidate the most appropriate channelrhodopsin and virus for chronic optogenetic excitation. For example, CatCh, a channelrhodopsin preferential to calcium entry, could be more appropriate for maturation of engineered cardiac tissue because calcium cycling induces hypertrophy in cardiac cells. ${ }^{15,54}$ Patch clamp could assess kinetics of different channelrhodopsin variants in this cell line to improve comparison of the variants. Also, a complementary study, similar to Tandon et 
al.'s study regarding electrical stimulation, focusing on viral dose and parameters of the conditioning protocol, such as pacing frequency, would be helpful. ${ }^{38}$ An improved viral dose could increase transfection efficiency; a higher transfection efficiency could improve pacing to ensure stimulation of the whole construct similar to complete field stimulation observed in electrical stimulation protocols. Possibly, complete stimulation of the whole construct simultaneously could improve construct maturity. However, greater transfection efficiency would require a balance with toxicity. Chronic optical pacing to d28 may increase C-OP construct maturity above non-paced d 28 construct. C-OP to d28 may overcome the paradox of extended culture's association with both maturity and massive cell loss. Also, to account for transfected endothelial cells and mural cells that might affect dose, we could stain for endothelial cells and mural cells, using an antibody for these cell types. Also, optimization of light intensity needed for optical excitation could reduce thermal effects and phototoxicity. ${ }^{51}$ In a future study, we could add a control group that undergoes light stimulation to directly assess the toxicity of our light system. With reduced toxicity, active stress of chronically optically stimulated constructs may improve. Furthermore, a cell viability assay would be beneficial to directly assess toxicity such as an MTT assay. We could assess toxicity of the virus itself by transfection of ECTs with a null, unpackaged, AAV virus. We could increase the number of d28 groups to further establish this group as a more mature experimental group. Finally, a more comprehensive gene and protein analysis could elucidate mechanistic insights of C-OP. This analysis could include protein phosphorylation studies to further define cell signaling pathways affected 
by C-OP. Further patch clamp studies could define the effect ChIEF activation has on other ion exchange channels at the $\mathrm{CM}$ membrane. 


\section{SUMMARY AND CONCLUSIONS}

The immature phenotype of ECTs may limit their therapeutic translation. Chronic conditioning protocols, such as, mechanical stretch, perfusion, and electrical stimulation induce tissue maturation. However, electrical stimulation can be invasive, produces toxic Faradaic byproducts, and has low spatiotemporal resolution. As an alternative, less invasive, less toxic, more precise method of chronic stimulation, we used optical excitation of heterologously expressed ChIEF light-sensitive ion channels.

Using an AAV packaged with a ChIEF, we successfully expressed light-sensitive ion channels in ECTs composed of human induced pluripotent derived cardiac cells. We successfully paced these constructs for 7 days, and this chronic stimulation induced electrophysiological maturation: improvements in MCR, hysteresis, KCND3 expression, and force-frequency relationships.

The research completed provides a novel chronic conditioning method, and, this conditioning method could possibly induce maturity in engineered cardiac tissues before regenerative treatments or drug testing. Further research in optogenetic applications to cardiac tissue engineering could improve this conditioning protocol to demonstrate improved efficacy compared to current non-optical conditioning protocols. 
A

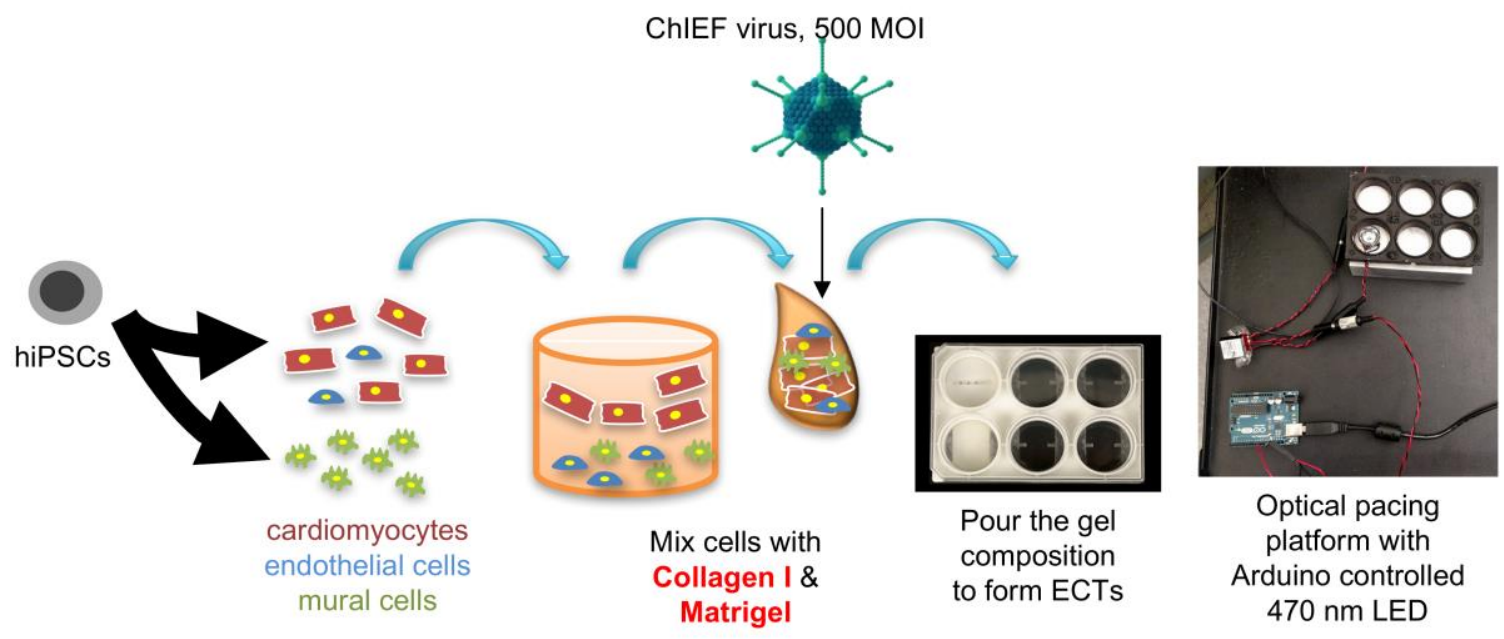

B

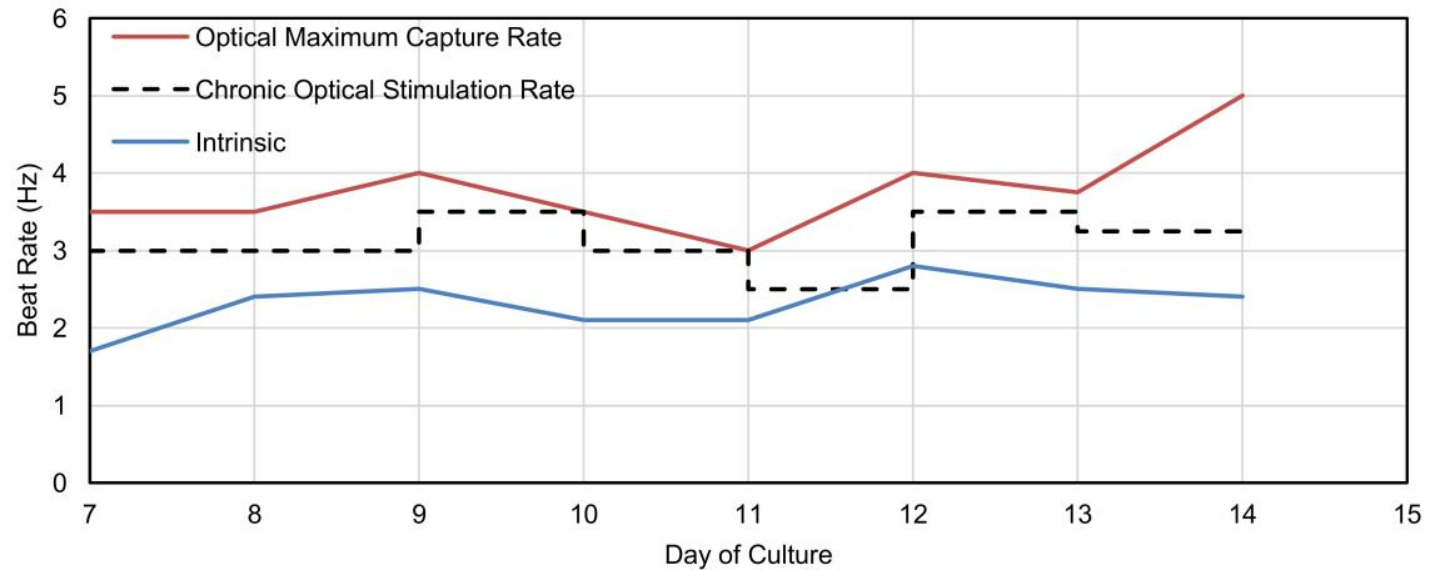

Figure 1. ECT formation and chronic optical pacing protocol. (A) ECTs were generated from a multi-component mixture of cardiomyocytes, endothelial cells, and mural cells, differentiated from h-iPSCs. Cells were mixed with culture medium, collagen I, and Matrigel. AAV1/2-CAG-oChIEF-tdTomato virus was then added to the gel mixture at 500 MOI. The gel mixture was poured into FlexCell TissueTrain wells deformed into a cylindrical mold by vacuum suction and anchored by nylon mesh tabs. The TissueTrain plate was placed on top of an Arduino-controlled LED platform for chronic optical pacing. ECTs were cultured at $37^{\circ} \mathrm{C}, 5 \% \mathrm{CO}_{2}$. (B) At culture day 7 , chronic optical stimulation was initiated using a pulsed $470 \mathrm{~nm}$ LED. A representative chronic optical stimulation protocol is shown. At each culture day, we measured the maximum capture rate and set the chronic optical stimulation rate $0.5 \mathrm{~Hz}$ below the maximal capture rate. 

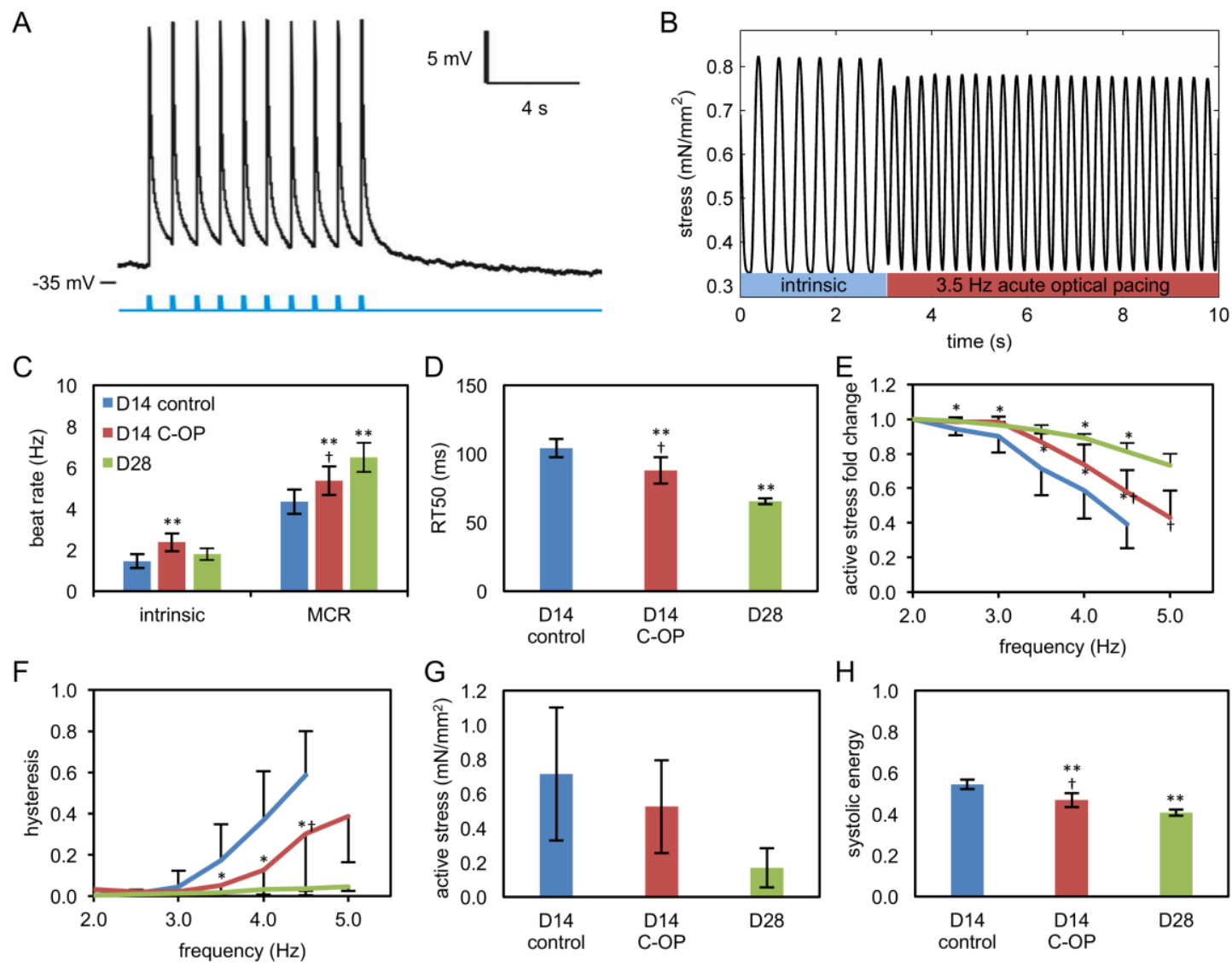

Figure 2. Impact of chronic optical pacing (C-OP) on functional maturation of $\mathrm{h}$-iPSC ECTs. (A) Representative patch clamp recording from a D14 h-iPSC-CM after ECT ChIEF transfection. Blue ticks indicate $470 \mathrm{~nm}$ light pulses. (B) Representative stress trace during acute optical pacing of a C-OP ECT. After 3 seconds of spontaneous beating, we began acute pacing with a $470 \mathrm{~nm} 3.5 \mathrm{~Hz}$ pulsed LED. (C) Mean intrinsic beat rate and maximal capture rate (MCR) for D14 control $(n=13)$, D14 C-OP $(n=15)$ and D28 $(n=2)$ ECTs. Intrinsic beat rate increased after $\mathrm{C}-\mathrm{OP}$ and $\mathrm{MCR}$ increased after $\mathrm{C}-\mathrm{OP}$ and prolonged culture to D28. (D) Mean RT50 AT $3 \mathrm{~Hz}$ acute electrical pacing decreased after C-OP and after prolonged culture to D28. (E) Stress-frequency curves showed a less negative relationship after C-OP or prolonged culture to D28. (F) Pacing rate dependent beat-to-beat hysteresis decreased after C-OP and was absent in D28 ECTs. (G) Mean active stress was similar in D14 control and C-OP ECTs and trended lower in D28 ECTs ( $p=0.08$ vs. D14 control). (H) Mean normalized systolic energy at $3 \mathrm{~Hz}$ acute electrical pacing decreased after C-OP and further decreased by D28. Error bars show standard deviations. See (C) for color legend. Results in (C-H) obtained with $5 \mathrm{~V}$ acute electrical field pacing. ${ }^{*} \mathrm{p}<0.05,{ }^{* *} \mathrm{p}<0.001 \mathrm{vs.}$ D14 control, $\uparrow p<0.05$ vs. D28. 

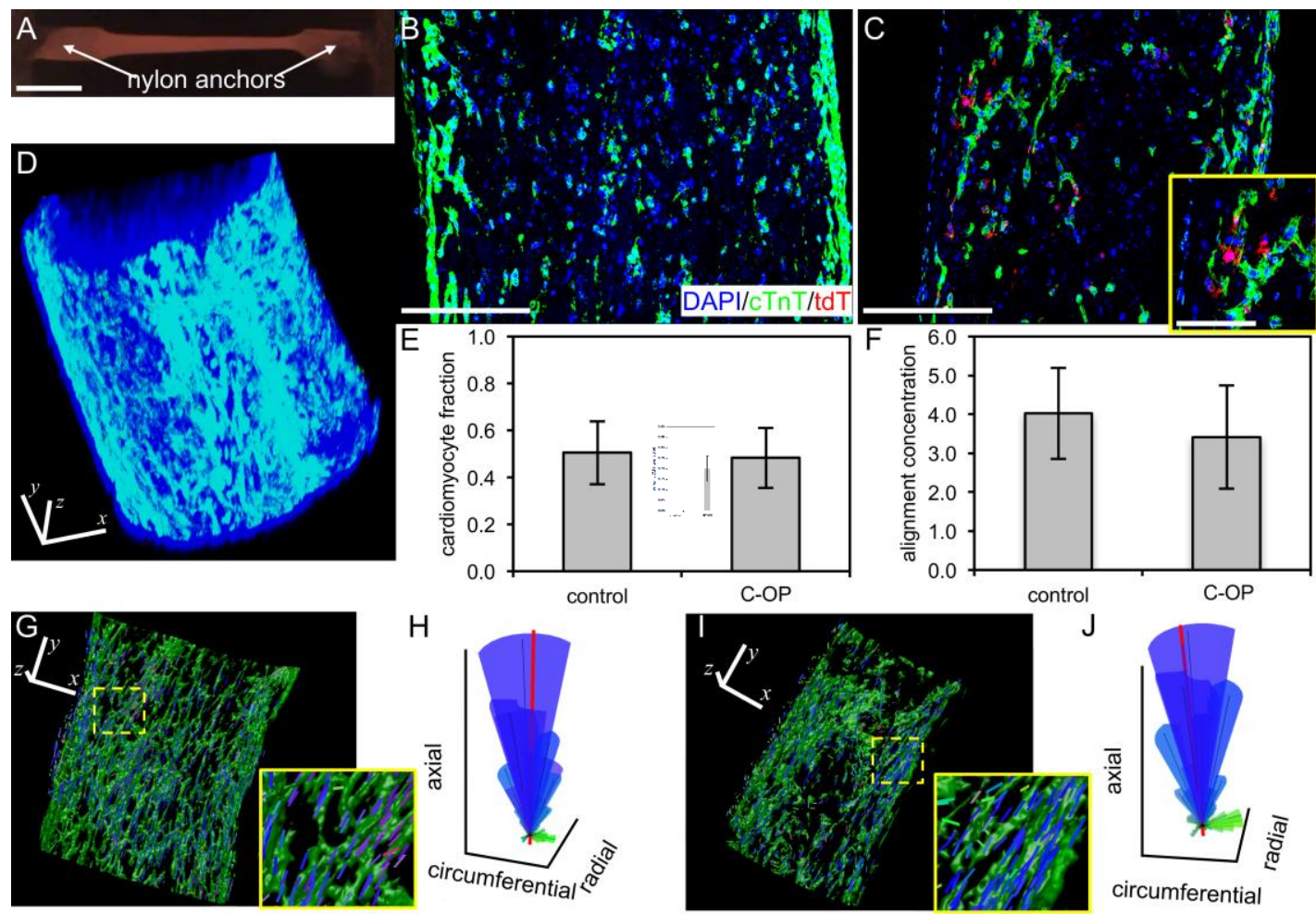

Figure 3. Impact of chronic optical pacing (C-OP) on structural maturation of $h$-iPSC ECTs. (A) Representative, linear and cylindrical h-iPSC ECT constrained the long axis ends by mesh nylon anchors. (B-C) Representative central paraffin sections of a (B) D14 control ECT showing 54\% cardiomyocytes (CM) and (C) D14 C-OP ECT showing 52\% CM and 13\% ChIEF-transfected cells. Nuclei were stained with DAPI (blue), CM with cTnT (green) and ChIEF-transfected cells with tdT (red). CM were predominantly located at the outer surface and the majority of transfected cells were CM. Inset in (C) shows higher magnification of double-positive cTnT and tdT cells. (D) Whole mount confocal image of a control D14 ECT. (E) Mean CM fraction showing mean CM transfection rate (inset). Mean CM fraction and (F) Mean alignment concentration $(\kappa)$ were similar in D14 control $(n=9)$ and C-OP $(n=10)$ h-iPSC ECTs. Error bars show standard deviations. (G-J) Representative local CM orientations within D14 (G) control and (I) C-OP h-iPSC ECTs. Color-coded lines indicate local CM orientation magnitudes in the circumferential (green), radial (red), and axial (blue) directions. For clarity, only a subset of the orientations is shown. Insets are $3 \mathrm{X}$ magnification of regions enclosed by the yellow dashed lines. ( $\mathbf{H}$ and J) Spherical histograms of local CM orientations. CM in both control and C-OP aligned parallel to the ECT long axis. The volume of each ray represents the relative count in each direction and the mean $\mathrm{CM}$ orientation is shown by the thick red line. $\kappa$ for the control sample $(\mathbf{H})$ was 3.89 while the C-OP sample (J) was 3.46. Scale bars: $5 \mathrm{~mm}(\mathbf{A}), 250 \mu \mathrm{m}$ (B, C, D, G, I), $100 \mu \mathrm{m}$ (C inset). Orientation axes also serve as scale bars in (D, G, I). 

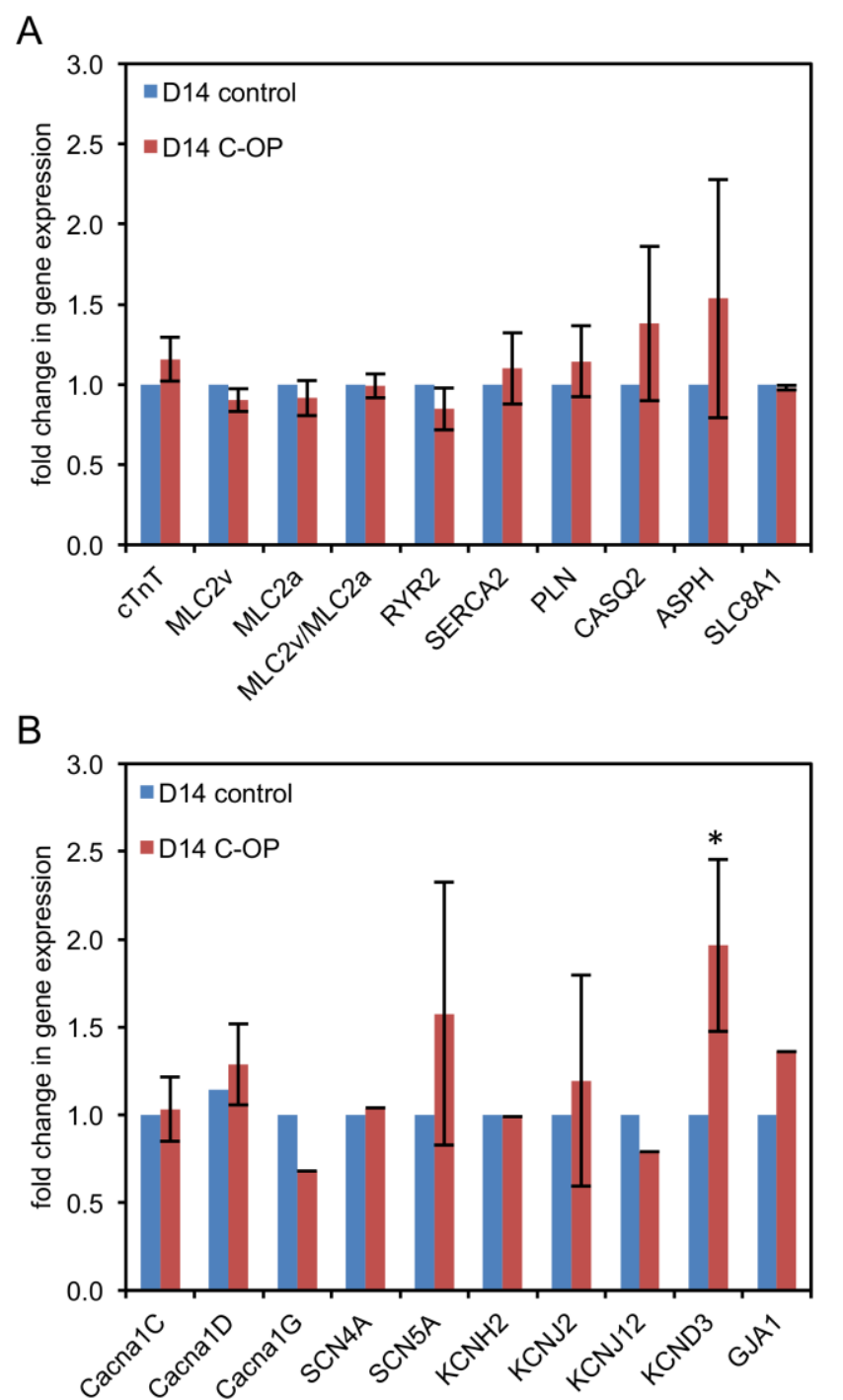

Figure 4. Quantitative PCR of genes encoding contractile proteins, $\mathrm{Ca}^{2+} \mathrm{Cycling}$ mechanisms, and ion channels in D14 control and C-OP ECTs. (A) Expression of genes related to cardiomyocyte contraction (cTnT, ventricular myosin light chain 2 [MLC2v], atrial MLC2 [MLC2a], and MLC2v/MLC2a ratio) and genes related to $\mathrm{Ca}^{2+}$ transients (ryanodine receptor 2 [RYR2], ATPase sarcoplasmic/endoplasmic reticulum $\mathrm{Ca}^{2+}$ transporting 2 [SERCA2], phospholamban [PLN], calsequestrin 2 [CASQ2], aspartate betahydroxylase [ASPH], and sodium/calcium exchanger 1 [SLC8A1]). C-OP did not produce significant differences in expression of contractile- or $\mathrm{Ca}^{2}{ }^{+}$-transient- related genes. (B) Expression of genes encoding sarcolemmal voltage-gated ion channels L-type $\mathrm{Ca}^{2+}$ (Cacna1C, Cacna1D), T-type Ca ${ }^{2+}$ (Cacna1G), $\mathrm{Na}^{+}$(SCN4A, SCN5A), and $\mathrm{K}^{+}$(KCNH2 [rapidly activating delayed rectifying], KCNJ2, KCNJ12 [inward rectifying], KCND3 [transient outward]) and connexin (GJA1). C-OP resulted in a significant increase in the expression of transient outward $\mathrm{K}^{+}$channel KCND3. Expression of all other ion channels and connexin were unchanged after C-OP. ${ }^{*} \mathrm{p}<0.05$. For each gene, $2-4$ biological experiments were conducted and qPCR was performed in triplicate for each biological sample. Error bars show standard deviations. 


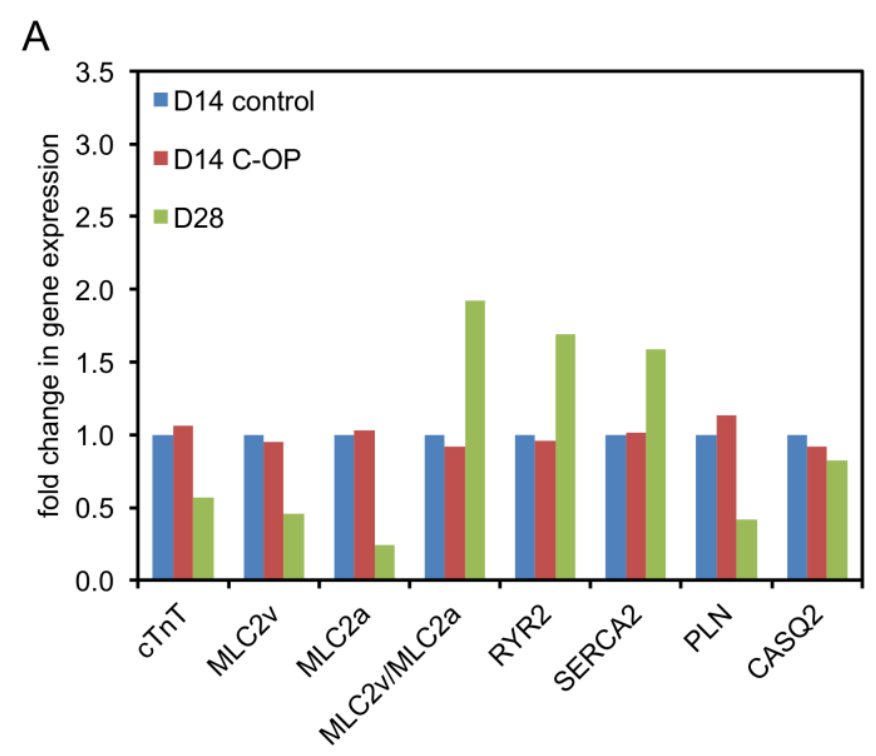

B

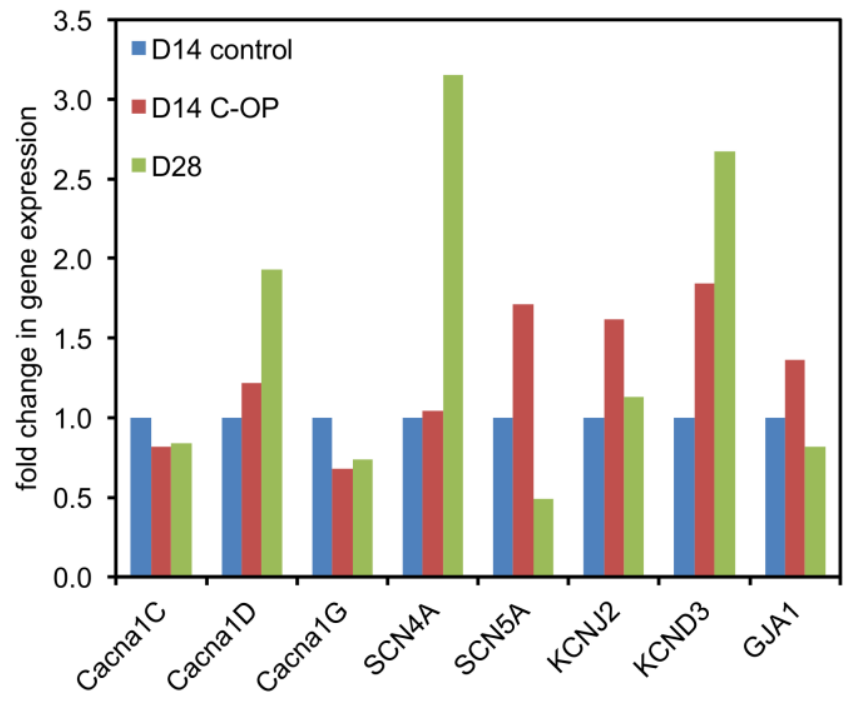

Figure 5. Quantitative PCR for a single biological experiment comparing D14 control, D14 C-OP, and D28 ECTs. (A) Expression of genes related to cardiomyocyte contraction (cTnT, ventricular myosin light chain 2 [MLC2v], atrial MLC2 [MLC2a], and MLC2v/MLC2a ratio) and genes related to $\mathrm{Ca}^{2+}$ transients (ryanodine receptor 2 [RYR2], ATPase sarcoplasmic/endoplasmic reticulum $\mathrm{Ca}^{2+}$ transporting 2 [SERCA2], phospholamban [PLN], and calsequestrin 2 [CASQ2]). Expression was similar for all genes in D14 control and C-OP, following the full qPCR study in Figure 3. The MLC2v/MLC2a was increased in the D28 sample, suggesting a more ventricular phenotype, while increased RYR2 and SERCA2 and decreased PLN suggest more mature $\mathrm{Ca}^{2+}$ handling. (B) Expression of genes encoding sarcolemmal voltage-gated ion channels L-type $\mathrm{Ca}^{2+}$ (Cacna1C, Cacna1D), T-type $\mathrm{Ca}^{2+}$ (Cacna1G), $\mathrm{Na}^{+}$(SCN4A, SCN5A), and $\mathrm{K}^{+}$(KCNJ2 [inward rectifying], KCND3 [transient outward]) and connexin (GJA1). The expression of transient outward $\mathrm{K}^{+}$channel KCND3 was increased in both the C-OP and D28 samples, suggesting a role for this channel in electrophysiological maturation during C-OP. L-type $\mathrm{Ca}^{2+}$ and $\mathrm{Na}^{+}$were also increased in the D28 sample, but did not show a similar increase in C-OP ECTs. 

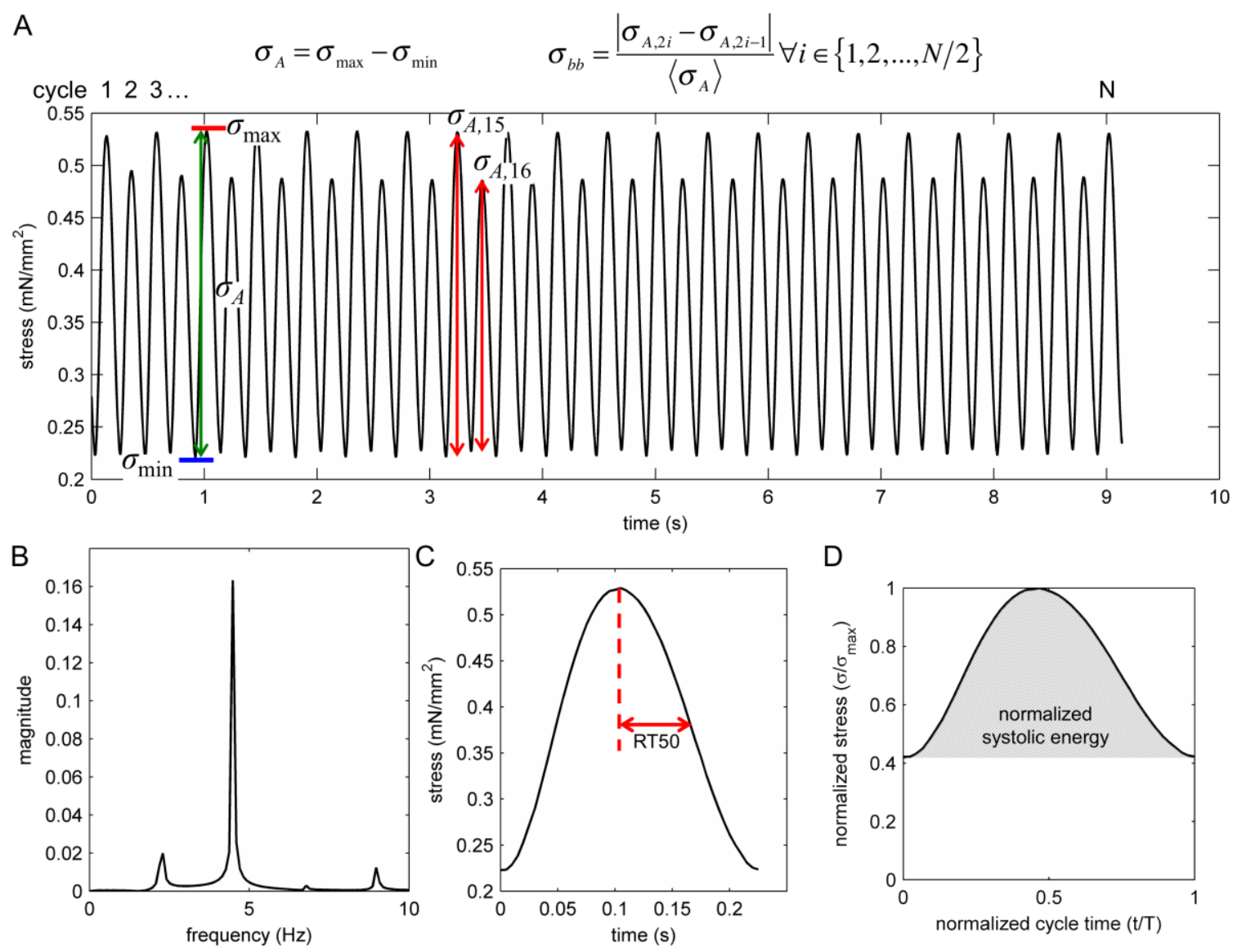

Figure S1. Analysis of ECT function. For this representative C-OP ECT at $4.5 \mathrm{~Hz}$ acute electrical pacing, mean $\sigma_{A}$ was $0.29 \mathrm{mN} / \mathrm{mm}^{2}, \sigma_{b b}$ was 0.17 , RT50 was $63 \mathrm{~ms}$, and normalized systolic energy was 0.53 . (A) Representative stress vs. time data. Force data was divided by ECT cross-sectional area to calculate stress. A cycle is defined as contraction followed by relaxation. The minimum stress before the onset of contraction is $\sigma_{\min }$ and the peak stress is $\sigma_{\max }$. Active stress, $\sigma_{A}$, is the difference between $\sigma_{\max }$ and $\sigma_{\min }$. Beat-to-beat hysteresis, $\sigma_{b b}$, is the magnitude of the difference between the $\sigma_{A}$ of an even-numbered cycle and the $\sigma_{A}$ of the preceding odd-numbered cycle, divided by the overall mean $\sigma_{A}$. (B) Fast Fourier transform (FFT) analysis of the stress vs. time signal. An acute electrical pacing frequency was considered captured if it matched the peak frequency of the FFT power spectrum. (C) RT50 calculated for cycle 1 in (A). RT50 is the time for stress to relax by $50 \%$ of $\sigma_{A}$ from $\sigma_{\max }$. (D) Normalized systolic energy calculated for cycle 1 in (A). Stress and cycle time were both normalized to a maximum value of 1 . The area of the shaded region under the stress-time curve is related to systolic energy during contraction. 

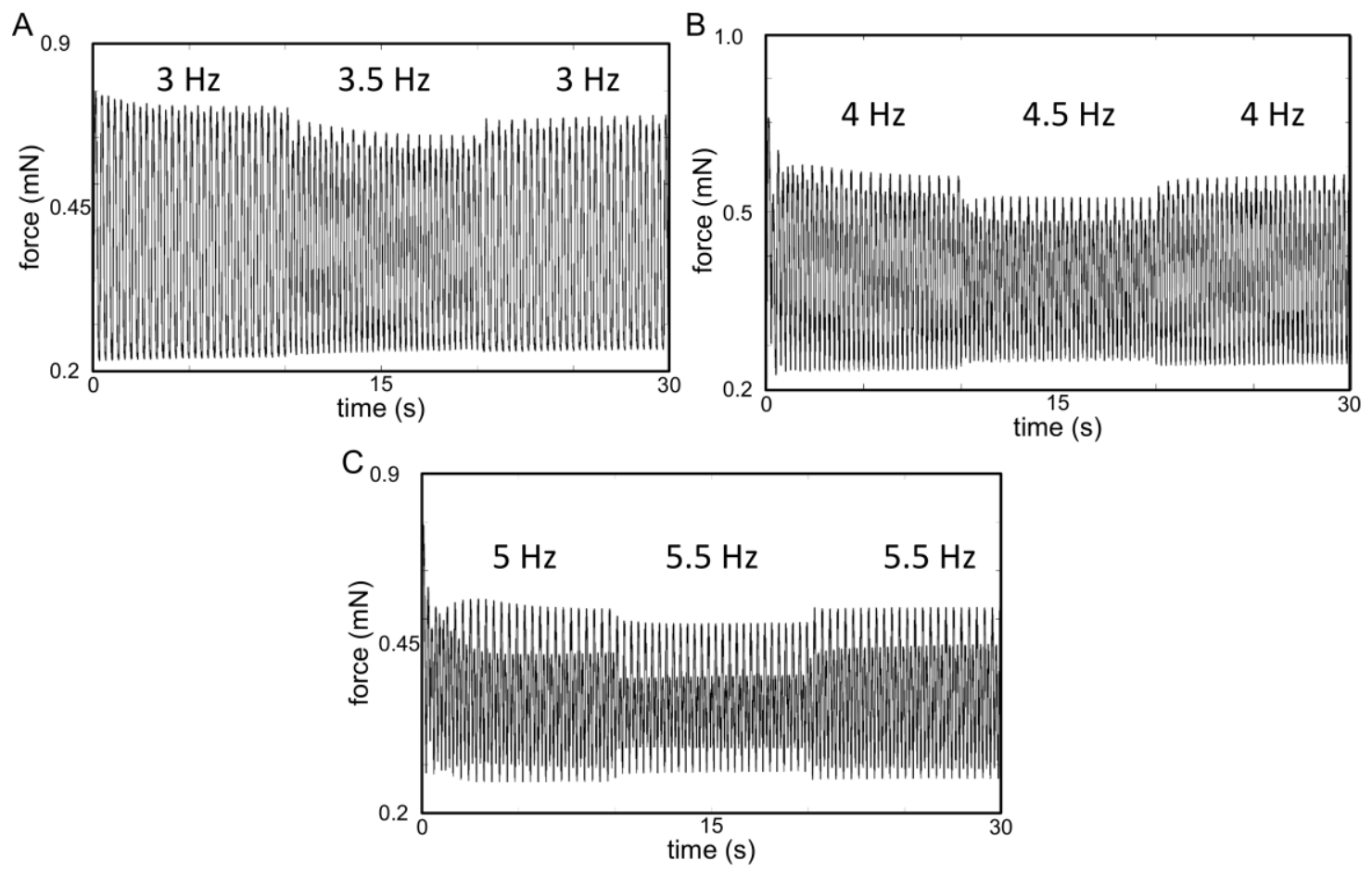

Figure S2. Representative force tracings at increasing pacing rates display decreasing active force and increasing beat-to-beat hysteresis. Note that there is a decrease in active force and increase in passive force with each increase in pacing frequency, consistent with the limited capacity for immature cardiac tissues to release and recapture calcium at higher pacing rates. Also note that there is a progressive increase in the beat to beat amplitude variation of active and passive force with each increase in pacing frequency, consistent with increased hysteresis. 


\section{REFERENCES}

1. Pagidipati, N.J. and Gaziano, T.A. Estimating Deaths from Cardiovascular Disease: A Review of Global Methodologies of Mortality Measurement. Circulation. 2013; 127: 749-756. doi: 10.1161/CIRCULATIONAHA.112.128413

2. Rasmussen, T.L, Raveendran, G., Zhang, J., and Garry, D.J. Getting to the Heart of Myocardial Stem Cells and Cell Therapy. Circulation. 2011; 123: 1771-1779. doi: 10.1161/CIRCULATIONHA.109.858019

3. Steinhauser, M.L. and Tee, R.T. Regeneration of the Heart. EMBO Molecular Medicine. 2011; 3: 701-712. doi: 10.1002/emmm.201100175

4. Sanganalmath, S.K. and Bolli, R. Cell Therapy for heart failure: A Comprehensive Overview of Experimental and Clinical Studies, Current Challenges, and Future Directions. Circulation Research. 2013; 113: 810-834. doi: 10. 1161/CIRCRESAHA.113.300219

5. Suuronen, E.J., Kuraitis, D., and Ruel, M. Improving Cell Engraftment with Tissue Engineering. Seminars in Thoracic and Cardiovascular Surgery. 2008; 2: 110-114. https://www-clinicalkey-com.echo.louisville I/\#!/content/playContent/1-s2.0S1043067908000609

6. Zimmerman, W.H., Melnychenko, I., Wasmeier, G., et al. Engineered heart tissue grafts improve systolic and diastolic function in infarcted rat hearts. Nature Medicine. 2006; 12: 452-458. doi: 10.1038/nm1394

7. Fujimoto, K.L., Clause, K.C., Liu, L.J., et al. Engineered fetal cardiac graft preserves its cardiomyocyte proliferation within postinfarcted myocardium and sustains cardiac function. Tissue Eng Part A. 2011; 17(5-6): 585-596. doi: 10.1089/ten.TEA.2010.0259.

8. Masumoto $\mathrm{H}$, Nakane $\mathrm{T}$, Tinney JP, et al. The myocardial regenerative potential of three-dimensional engineered cardiac tissues composed of multiple human iPS cellderived cardiovascular cell lineages. Scientific Reports. 2016;6:29933. doi:10.1038/srep29933. 
9. Hirt, M.N., Hansen, A., and Eschenhagen, T. Cardiac Tissue Engineering: State of the art. Circulation Research. 2014; 114(2): 354-367. doi:

10.1161/CIRCRESAHA.114.3000522

10. Maidhof R, Tandon N, Lee EJ, et al. Biomimetic perfusion and electrical stimulation applied in concert improved the assembly of engineered cardiac tissue. Journal of Tissue Engineering and Regenerative Medicine. 2011;6(10). doi:10.1002/term.525

11. Stoppel WL, Kaplan DL, Black LD. Electrical and mechanical stimulation of cardiac cells and tissue constructs. Advanced Drug Delivery Reviews. 2016;96:135-155. doi:10.1016/j.addr.2015.07.009.

12. Hernandez, D., Millard, R., Sivakumaran, P., et al. Electrical Stimulation Promotes Cardiac Differentiation of Human Induced Pluripotent Stem Cells. Stem Cells Int. 2016; 2016: 1718041. doi: 10.11155/2016/1718041.

13. Balint R, Cassidy NJ, Cartmell SH. Electrical Stimulation: A Novel Tool for Tissue Engineering. Tissue Engineering Part B: Reviews. 2013;19(1):48-57. doi:10.1089/ten.teb.2012.0183.

14. Serena E, Figallo E, Tandon N, et al. Electrical stimulation of human embryonic stem cells: Cardiac differentiation and the generation of reactive oxygen species. Experimental Cell Research. 2009;315(20):3611-3619.

15. McDonough PM, Glembotski CC. Induction of atrial natriuretic factor and myosin light chain-2 gene expression in cultured ventricular myocytes by electrical stimulation of contraction. Journal of Biological Chemistry. 1992;267(17):1166511668.

16. McDonough PM, Stella SL, Glembotski CC. Involvement of cytoplasmic calcium and protein kinases in the regulation of atrial natriuretic factor secretion by contraction rate and endothelin. Journal of Biological Chemistry. 1994;269(13):9466-9472.

17. Mcdonough PM, Hanford DS, Sprenkle AB, Mellon NR, Glembotski CC. Collaborative Roles for c-Jun N-terminal Kinase, c-Jun, Serum Response Factor, and Sp1 in Calciumregulated Myocardial Gene Expression. Journal of Biological Chemistry. 1997;272(38):24046-24053. doi:10.1074/jbc.272.38.24046.

18. Xia Y, Buja LM, Mcmillin JB. Change in Expression of Heart Carnitine Palmitoyltransferase I Isoforms with Electrical Stimulation of Cultured Rat Neonatal Cardiac Myocytes. Journal of Biological Chemistry. 1996;271(20):12082-12087. doi:10.1074/jbc.271.20.12082. 
19. Xia Y, Buja LM, Scarpulla RC, Mcmillin JB. Electrical stimulation of neonatal cardiomyocytes results in the sequential activation of nuclear genes governing mitochondrial proliferation and differentiation. Proceedings of the National Academy of Sciences. 1997;94(21):11399-11404. doi:10.1073/pnas.94.21.11399.

20. Xia Y, Buja LM, Mcmillin JB. Activation of the Cytochrome c Gene by Electrical Stimulation in Neonatal Rat Cardiac Myocytes: ROLE OF NRF-1 AND c-Jun. Journal of Biological Chemistry. 1998;273(20):12593-12598. doi:10.1074/jbc.273.20.12593.

21. Xia Y, Mcmillin JB, Lewis A, et al. Electrical Stimulation of Neonatal Cardiac Myocytes Activates the NFAT3 and GATA4 Pathways and Up-regulates the Adenylosuccinate Synthetase 1 Gene. Journal of Biological Chemistry. 2000;275(3):1855-1863. doi:10.1074/jbc.275.3.1855.

22. Kaye D, Pimental D, Prasad S, et al. Role of transiently altered sarcolemmal membrane permeability and basic fibroblast growth factor release in the hypertrophic response of adult rat ventricular myocytes to increased mechanical activity in vitro. Journal of Clinical Investigation. 1996;97(2):281-291. doi:10.1172/jci118414.

23. Chan Y-C, Ting S, Lee $\mathrm{Y}-\mathrm{K}$, et al. Electrical Stimulation Promotes Maturation of Cardiomyocytes Derived from Human Embryonic Stem Cells. Journal of Cardiovascular Translational Research. 2013;6(6):989-999. doi:10.1007/s12265-0139510-z.

24. Maxwell JT, Wagner MB, Davis ME. Electrically Induced Calcium Handling in Cardiac Progenitor Cells. Stem Cells International. 2016;2016:1-11. doi:10.1155/2016/8917380.

25. Rackauskas G, Saygili E, Rana OR, et al. Subthreshold High-Frequency Electrical Field Stimulation Induces VEGF Expression in Cardiomyocytes. Cell Transplantation. 2015;24(8):1653-1659. doi:10.3727/096368914x682783.

26. Park $H$, Bhalla R, Saigal $R$, et al. Effects of electrical stimulation in C2C12 muscle constructs. Journal of Tissue Engineering and Regenerative Medicine. 2008;2(5):279287. doi:10.1002/term.93.

27. Pedrotty DM. Engineering skeletal myoblasts: roles of three-dimensional culture and electrical stimulation. AJP: Heart and Circulatory Physiology. 2004;288(4). doi:10.1152/ajpheart.00610.2003.

28. Wang B, Wang G, To F, et al. Myocardial Scaffold-Based Cardiac Tissue Engineering: Application of Coordinated Mechanical and Electrical Stimulations. Langmuir. 2013;29(35):11109-11117. doi:10.1021/la401702w. 
29. Mooney E, Mackle JN, Blond DJ-P, et al. The electrical stimulation of carbon nanotubes to provide a cardiomimetic cue to MSCs. Biomaterials. 2012;33(26):61326139. doi:10.1016/j.biomaterials.2012.05.032.

30. Tandon N, Goh B, Marsano A, et al. Alignment and elongation of human adiposederived stem cells in response to direct-current electrical stimulation. 2009 Annual International Conference of the IEEE Engineering in Medicine and Biology Society. 2009. doi:10.1109/iembs.2009.5333142.

31. Pavesi A, Soncini M, Zamperone A, et al. Electrical conditioning of adipose-derived stem cells in a multi-chamber culture platform. Biotechnology and Bioengineering. 2014;111(7):1452-1463. doi:10.1002/bit.25201.

32. Radisic $M$, Park $H$, Shing $H$, et al. Functional assembly of engineered myocardium by electrical stimulation of cardiac myocytes cultured on scaffolds. Proceedings of the National Academy of Sciences. 2004;101(52):18129-18134.

doi:10.1073/pnas.0407817101.

33. Au HTH, Cheng I, Chowdhury MF, Radisic M. Interactive effects of surface topography and pulsatile electrical field stimulation on orientation and elongation of fibroblasts and cardiomyocytes. Biomaterials. 2007;28(29):4277-4293. doi:10.1016/j.biomaterials.2007.06.001.

34. Park H, Larson BL, Kolewe ME, Vunjak-Novakovic G, Freed LE. Biomimetic scaffold combined with electrical stimulation and growth factor promotes tissue engineered cardiac development. Experimental Cell Research. 2014;321(2):297-306. doi:10.1016/j.yexcr.2013.11.005.

35. Richards DJ, Tan Y, Coyle R, et al. Nanowires and Electrical Stimulation Synergistically Improve Functions of h-iPSC Cardiac Spheroids. Nano Letters. 2016;16(7):4670-4678. doi:10.1021/acs.nanolett.6b02093.

36. Ahadian S, Yamada S, Ramón-Azcón J, et al. Hybrid hydrogel-aligned carbon nanotube scaffolds to enhance cardiac differentiation of embryoid bodies. Acta Biomaterialia. 2016;31:134-143. doi:10.1016/j.actbio.2015.11.047.

37. Tandon N, Marsano A, Cannizzaro C, Voldman J, Vunjak-Novakovic G. Design of electrical stimulation bioreactors for cardiac tissue engineering. 2008 30th Annual International Conference of the IEEE Engineering in Medicine and Biology Society. 2008. doi:10.1109/iembs.2008.4649983.

38. Tandon N, Marsano A, Maidhof R, Wan L, Park H, Vunjak-Novakovic G. Optimization of electrical stimulation parameters for cardiac tissue engineering. Journal of Tissue Engineering and Regenerative Medicine. 2011;5(6). doi:10.1002/term.377. 
39. Chiu LL, Iyer RK, King J-P, Radisic M. Biphasic Electrical Field Stimulation Aids in Tissue Engineering of Multicell-Type Cardiac Organoids. Tissue Engineering Part A. 2011;17(11-12):1465-1477. doi:10.1089/ten.tea.2007.0244.

40. Pietronave S, Zamperone A, Oltolina F, et al. Monophasic and Biphasic Electrical Stimulation Induces a Precardiac Differentiation in Progenitor Cells Isolated from Human Heart. Stem Cells and Development. 2014;23(8):888-898. doi:10.1089/scd.2013.0375.

41. Lasher RA, Pahnke AQ, Johnson JM, Sachse FB, Hitchcock RW. Electrical stimulation directs engineered cardiac tissue to an age-matched native phenotype. Journal of Tissue Engineering. 2012;3(1). doi:10.1177/2041731412455354.

42. Hirt MN, Boeddinghaus J, Mitchell A, et al. Functional improvement and maturation of rat and human engineered heart tissue by chronic electrical stimulation. Journal of Molecular and Cellular Cardiology. 2014;74:151-161.

doi:10.1016/j.yjmcc.2014.05.009.

43. Nunes SS, Miklas JW, Liu J, et al. Biowire: a platform for maturation of human pluripotent stem cell-derived cardiomyocytes. Nature Methods. 2013;10(8):781787. doi:10.1038/nmeth.2524.

44. Godier-Furnémont AF, Tiburcy M, Wagner $E$, et al. Physiologic force-frequency response in engineered heart muscle by electromechanical stimulation. Biomaterials. 2015;60:82-91. doi:10.1016/j.biomaterials.2015.03.055.

45. Williams JCAC, Entcheva E. Optogenetic versus Electrical Stimulation of Human Cardiomyocytes: Modeling Insights. Biophysical Journal. 2015;108(8):1934-1945. doi:10.1016/j.bpj.2015.03.032.

46. Bruegmann T, Malan D, Hesse M, et al. Optogenetic control of heart muscle in vitro and in vivo. Nature Methods. 2010;7(11):897-900. doi:10.1038/nmeth.1512.

47. Abilez OJ. Cardiac optogenetics. 2012 Annual International Conference of the IEEE Engineering in Medicine and Biology Society. 2012. doi:10.1109/embc.2012.6346197.

48. Deisseroth K. Optogenetics. Nature Methods. 2010;8(1):26-29. doi:10.1038/nmeth.f.324.

49. Abilez OCAJ, Wong J, Prakash R, Deisseroth K, Zarins CK, Kuhl E. Multiscale Computational Models for Optogenetic Control of Cardiac Function. Biophysical Journal. 2011;101(6):1326-1334. doi:10.1016/j.bpj.2011.08.004. 
50. Boyden ES, Zhang F, Bamberg E, Nagel G, Deisseroth K. Millisecond-timescale, genetically targeted optical control of neural activity. Nature Neuroscience. 2005;8(9):163-1268. doi:10.1038/nn1525.

51. Jia Z, Valiunas V, Lu Z, et al. Stimulating Cardiac Muscle by Light: Cardiac Optogenetics by Cell Delivery. Circulation: Arrhythmia and Electrophysiology. 2011;4(5):753-760. doi:10.1161/circep.111.964247.

52. Lin JY. A user's guide to channelrhodopsin variants: features, limitations and future developments. Experimental Physiology. 2010;96(1):19-25.

doi:10.1113/expphysiol.2009.051961.

53. Chow BY, Han X, Boyden ES. Genetically encoded molecular tools for light-driven silencing of targeted neurons. Progress in Brain Research Optogenetics: Tools for Controlling and Monitoring Neuronal Activity. 2012:49-61. doi:10.1016/b978-0-44459426-6.00003-3.

54. Bingen BO, Engels MC, Schalij MJ, et al. Light-induced termination of spiral wave arrhythmias by optogenetic engineering of atrial cardiomyocytes. Cardiovascular Research. 2014;104(1):194-205. doi:10.1093/cvr/cvu179.

55. Nyns EC, Kip A, Bart Cl, et al. Optogenetic termination of ventricular arrhythmias in the whole heart: towards biological cardiac rhythm management. European Heart Journal. 2016. doi:10.1093/eurheartj/ehw574.

56. Arrenberg AB, Stainier DYR, Baier H, Huisken J. Optogenetic Control of Cardiac Function. Science. 2010;330(6006):971-974. doi:10.1126/science.1195929.

57. Klimas A, Yu J, Ambrosi CM, Williams JC, Bien H, Entcheva E. OptoDyCE: Automated System for High-Throughput All-Optical Dynamic Cardiac Electrophysiology. 2015. doi:10.1101/023572.

58. Dempsey GT, Werley CA. Optogenetic Approach to Cardiotoxicity Screening: Simultaneous Voltage and Calcium Imaging Under Paced Conditions. Methods in Pharmacology and Toxicology Stem Cell-Derived Models in Toxicology. 2016:109134. doi:10.1007/978-1-4939-6661-5_6.

59. Abilez OJ. Optogenetic LED array for perturbing cardiac electrophysiology. 2013 35th Annual International Conference of the IEEE Engineering in Medicine and Biology Society (EMBC). 2013. doi:10.1109/embc.2013.6609826.

60. Sakar MS, Neal D, Boudou T, et al. Formation and optogenetic control of engineered 3D skeletal muscle bioactuators. Lab on a Chip. 2012;12(23):4976.

doi:10.1039/c2lc40338b. 
61. Chan V, Neal DM, Uzel SGM, Kim H, Bashir R, Asada HH. Fabrication and characterization of optogenetic, multi-strip cardiac muscles. Lab Chip. 2015;15(10):2258-2268. doi:10.1039/c5lc00222b.

62. Nussinovitch U, Shinnawi R, Gepstein L. Modulation of cardiac tissue electrophysiological properties with light-sensitive proteins. Cardiovascular Research. 2014;102(1):176-187. doi:10.1093/cvr/cvu037.

63. Burton RAB, Klimas A, Ambrosi CM, et al. Optical control of excitation waves in cardiac tissue. Nature Photonics. 2015;9(12):813-816.

doi:10.1038/nphoton.2015.196.

64. Nussinovitch $U$, Gepstein L. Optogenetics for in vivo cardiac pacing and resynchronization therapies. Nature Biotechnology. 2015;33(7):750-754. doi:10.1038/nbt.3268.

65. Zaglia T, Pianca N, Borile G, et al. Optogenetic determination of the myocardial requirements for extrasystoles by cell type-specific targeting of Channelrhodopsin- 2 . Proceedings of the National Academy of Sciences. 2015;112(32). doi:10.1073/pnas.1509380112.

66. Bickford ME, Zhou N, Krahe TE, Govindaiah G, Guido W. Retinal and Tectal "DriverLike" Inputs Converge in the Shell of the Mouse Dorsal Lateral Geniculate Nucleus. Journal of Neuroscience. 2015;35(29):10523-10534. doi:10.1523/jneurosci.337514.2015.

67. Zsebo K, Yaroshinsky A, Rudy JJ, et al. Long-Term Effects of AAV1/SERCA2a Gene Transfer in Patients With Severe Heart Failure Novelty and Significance. Circulation Research. 2013;114(1):101-108. doi:10.1161/circresaha.113.302421.

68. Guan X, Wang Z, Czerniecki S, et al. Use of adeno-associated virus to enrich cardiomyocytes derived from human stem cells. Human Gene Therapy Clinical Development. 2015:150715074418003. doi:10.1089/hum.2015.052.

69. Pinnell J, Turner S, Howell S. Cardiac muscle physiology. Continuing Education in Anaesthesia, Critical Care \& Pain. 2007;7(3):85-88. doi:10.1093/bjaceaccp/mkm013.

70. Nakane T, Masumoto H, Tinney J, et al. Impact of Cell Composition and Geometry on Human Induced Pluripotent Stem Cells-Derived Engineered Cardiac Tissue. Nature Scientific Reports. 2017;7:45641. doi:10.1038/srep45641

71. Sato D. Spatially Discordant Alternans in Cardiac Tissue: Role of Calcium Cycling. Circulation Research. 2006;99(5):520-527. doi:10.1161/01.res.0000240542.03986.e7. 
72. Holubarsch Ch. Acute and chronic changes of myocardial energetics in the mammalian and human heart. In: Jacob R, ed. Cardiac Energetics: Basic Mechanisms and Clinical Implications. Darmstadt: Steinkopff; 2013: 386

73. Sartiani L, Bettiol E, Stillitano F, Mugelli A, Cerbai E, Jaconi ME. Developmental Changes in Cardiomyocytes Differentiated from Human Embryonic Stem Cells: A Molecular and Electrophysiological Approach. Stem Cells. 2007;25(5):1136-1144. doi: 10. 1634/stemcells.2006-0466.

74. Hoppe UC, Marban E, Johns DC. Molecular dissection of cardiac repolarization by in vivo Kv4.3 gene transfer. Journal of Clinical Investigation. 2000;105(8):1077-1084. doi:10.1172/jci8757

75. Pasquier J, Gupta R, Rioult D, et al. Coculturing with endothelial cells promotes in vitro maturation and electrical coupling of human embryonic stem cell-derived cardiomyocytes. The Journal of Heart and Lung Transplantation. 2017. doi: 10.1016/j.healun.2017.01.001.

76. Zhang S. Cui N, Wu Y, Zhong W, Johnson CM, Jiang C. Optogenetic intervention to the vascular endothelium. Vascular Pharmacology. 2015;74:7122-129.

doi:10.1016/j.vph.2015.05.009 


\section{DEPARTMENT OF ENVIRONMENTAL HEALTH AND SAFETY}

University of Louisville

1800 Arthur St

Louisville KY 40208-2729

\section{UNIVERSITY of LOUISVILLE。}

March 07, 2016

RE: IBC Registration \#IBC 16-105: New human iPSC-derived cardiomyocytes for cardiac repair Effective 03/07/2016; Expires 03/06/2021

Dear Dr. Keller, The above registration was reviewed and approved by the Institutional Biosafety Committee. Institutional Biosafety Committee approval is granted for a period of five (5) years. During the approval period, it is the responsibility of the Principal Investigator to notify the IBC of any changes (via Modification Request in iRIS):

- Addition of Risk Group 2 and above agents

- Addition of Personnel working on the protocol

- Change of location of research facilities

- Significant modification of methods, specifically to include aerosol generating equipment (e.g. cell counter)

A new application must be submitted before the end of the five-year period as outlined in the IBC Application/Registration Instructions that can be found on the DEHS web site at https://louisville.edu/dehs/biosafety/institutional-biosafety-committee-ibc.html.

It is the responsibility of the Principal Investigator to ensure that all individuals performing activities under this protocol maintain required training certifications for the duration of the approval period. Specifically, if you are working with human or primate source materials, annual bloodborne pathogen training is required. Biosafety, Bloodborne Pathogens and other online training courses are offered through the Department of Environmental Health and Safety (via Bioraft at:

https://louisville.bioraft.com). Any agent / organism / lab specific training must be provided by the Principal Investigator or designee.

If you have any questions, please contact the Biosafety Program, or myself, in the Department of Environmental Health and Safety at 852-6670.

Sincerely,

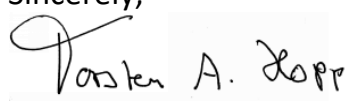

Torsten A. Hopp, Ph.D.

Biosafety Officer

Department of Environmental Health and Safety 


\section{CURRICULUM VITAE}

Marc Dwenger

Date of Birth: January 15, 1991

Email Address: MODWEN01@cardmail.louisville.edu

EDUCATION

Master of Science, Pharmacology and Toxicology

2018 (anticipated)

Bachelor of Arts, Biology

Simpson College, Indianola, IA

2013

HONORS

2009 Great Ape Studies Scholarship

2009 Presidential Scholarship

2010 Floyd S. Doft Scholarship

2010 Floyd S. Doft Science Honor Award

2011 Dr G.A.\& Helen Grant Scholarship

2011 CRC Press Chemistry Achievement Award

2013 Summa Cum Laude

2009-2013 resident or Dean's List (8 semesters)

$\underline{\text { PROFESSIONAL SOCIETIES AND MEMBERSHIPS }}$

2012 Epsilon Sigma Academic Honor Society,

2012 Sigma Pi Sigma Physics Honor Society

2011 Beta Beta Beta Biology Honors Society 


\section{PUBLICATIONS}

Kowalski WJ, Yuan F, Nakane T, Masumoto H, Dwenger M, Ye F, Tinney JP, and Keller BB (2016) Quantification of cardiomyocyte alignment from 3D confocal microscopy of engineered tissue. Microscopy and Microanalysis. (Revised, Under Review).

\section{PRESENTATIONS}

04/2015

Research Seminar - "Evidence for iKr potassium channel maturation in chick ECT by E4031 block" University of Louisville Seminar in Pharmacology and Toxicology Louisville, KY

10/2015

Poster Presentation - "Design and Fabrication of an Optically Activated Engineered Cardiac Tissue"

Research! Louisville

Louisville, KY

10/2016

Poster Presentation - "Chronic OP of Human iPSC-derived Engineered Cardiac Tissues" Research! Louisville Louisville, KY 